\title{
Le travail de management en tant qu'activité de cadrage et de recadrage du contexte des transactions coopératives
}

Management as the activity of framing or reframing the context of cooperative transactions

\section{Manuel Zacklad}

\section{OpenEdition}

Journals

Édition électronique

URL : http://journals.openedition.org/activites/650

DOI : 10.4000/activites.650

ISSN : $1765-2723$

Éditeur

ARPACT - Association Recherches et Pratiques sur les ACTivités

\section{Référence électronique}

Manuel Zacklad, «Le travail de management en tant qu'activité de cadrage et de recadrage du contexte des transactions coopératives ", Activités [En ligne], 10-1 | Avril 2013, mis en ligne le 15 avril 2013, consulté le 19 avril 2019. URL : http://journals.openedition.org/activites/650 ; DOI : 10.4000/ activites.650

\section{(c) $\oplus \Theta \Theta$}

Activités est mis à disposition selon les termes de la licence Creative Commons Attribution - Pas d'Utilisation Commerciale - Pas de Modification 4.0 International. 


\title{
Le travail de management en tant qu'activité de cadrage et de recadrage du contexte des transactions coopératives
}

\author{
Manuel Zacklad \\ Conservatoire National des Arts et Métiers - Laboratoire DICEN EA 4420 \\ CNAM - CITS - 16SP30 - 292, rue Saint Martin - 75141 Paris Cedex 03 \\ manuel.zacklad@cnam.fr
}

\begin{abstract}
Management as the activity of framing or reframing the context of cooperative transactions. In this paper we shall describe managerial work using the "Semiotics of Cooperative Transactions" theory. Managerial work will be considered as an activity of framing cooperative transactions which may involve specialized personnel, managers or executives, but more generally all the employees of the organization. This work is sometimes called regulatory work (Reynaud, 1989), organizational work (de Terssac, 2002), articulation work (Strauss, 1993) or meta-functional activity (Falzon, 1994). We start by presenting the main categories of the semiotic of cooperative transactions which positions itself as a framework for the analysis of action and practice at a "meso" level grounded in the pragmatist thought tradition. This level of understanding should facilitate the mediation between analysis at the micro level of situated interactions and the macro level of the organizational processes. To make this link we rely on the pragmatist notions of situation and context. We illustrate these concepts through a case study involving issues of change management in a university aiming to introduce strengthened managerial control in the context of the reform of French universities. This case will be interpreted in terms of framing, conflict framing, reframing of the transactional context as being part of the management of change and resistance to change.
\end{abstract}

\section{KEYWORDS}

activity analysis, managerial work, transaction, semiotic, pragmatism

\section{1.- Introduction}

Dans cet article nous nous appuierons sur la Sémiotique des Transactions Coopératives (Zacklad, 2000, 2005, 2010a) pour analyser le travail management que nous définirons comme une activité de cadrage des transactions coopératives qui peut concerner des opérateurs spécialisés, les managers ou les cadres, mais qui plus généralement concerne tous les membres de l'entreprise en tant qu'ils s'impliquent un travail de management, parfois qualifié de travail de régulation (Reynaud, 1989), travail d'organisation (de Terssac, 2002), travail d'articulation (Strauss, 1993) ou d'activité méta-fonctionnelle (Falzon, 1994). Notons d'emblée que notre acception du management recouvre y compris des enjeux de cadrage technique de l'activité. Selon une perspective strictement cognitive, dont nous proposerons ici un dépassement, ces activités relèvent en partie des activités de planification que l'on peut appréhender selon les 
deux dimensions de l'anticipation temporelle et de l'abstraction (Hoc, 1987). Par rapport à ces courants de recherche, la sémiotique des transactions coopératives nous permettra d'adopter une approche plus intégrée, réconciliant les dimensions sociales et cognitives, mais également plus ouverte aux sciences de gestion et aux dimensions politiques, notamment par l'intégration explicite de problématiques stratégiques. Ce faisant, nous serons amené à porter une attention particulière au travail immatériel, au sens donné à ce terme par les économistes, c'est-à-dire au travail dont le produit n'est pas directement mesurable, comme c'est le cas de l'essentiel du travail serviciel ou du travail des métiers tertiaires dans l'industrie. Pour une large part, ce travail est d'essence communicationnelle (Zacklad, 2012a).

Nous commencerons par présenter les principales catégories de la sémiotique des transactions coopérative qui se positionne comme un cadre d'analyse de l'action et de la pratique au niveau «méso », encore en développement, mais comparable à ceux proposés dans d'autres théories en sciences cognitives, en psychologie, en sociologie, en sciences de l'information et de la communication, voire dans certaines approches de l'anthropologie ou de la philosophie des techniques. Parmi ces théories, on peut établir une distinction entre celles qui s'en tiennent à l'introduction de concepts heuristiques à un haut niveau d'abstraction, mêmes si elles s'appuient sur des études de terrains assez micro et celles qui visent à proposer des langages de description de l'activité systématiques ${ }^{1}$ comme nous cherchons à le faire. Ces langages visent à fournir aux chercheurs et aux praticiens des aides méthodologiques permettant l'analyse des situations selon les principes dégagés au plan théorique. Les deux approches sont complémentaires et s'alimentent mutuellement.

Pour faire le lien entre l'analyse micro des interactions médiatisées et les processus d'organisation et de création de valeur appréhendés à des niveaux plus macro, nous insisterons plus particulièrement dans cet article sur l'approche pragmatiste des notions de situation et de contexte qui nous permettront de rendre compte de cette dimension essentielle de l'action collective que sont les activités de cadrage. Nous illustrerons ces notions par la présentation d'un cas correspondant à la problématique d'une conduite de changement dans un milieu universitaire visant à introduire un contrôle gestionnaire renforcé inscrit dans la nouvelle loi sur la réforme des universités françaises. Ce cas sera interprété en termes de cadrage, de conflit de cadrage, de recadrage du contexte transactionnel contribuant à la conduite du changement et à la résistance au changement.

\section{2.- Première approche des transactions coopératives}

\section{1.- Première approche des transactions}

La sémiotique des transactions coopératives place au cœur de son analyse le renouvellement théorique du concept de transaction (voir aussi Renault, 2004, et dans ce numéro ainsi que Simpson 2013, Bidet, Boutet, \& Chave, 2013) en définissant les «transactions coopératives » comme un cadre privilégié pour une description analytique des activités individuelles et collectives. Le concept de transaction, que nous empruntons à Dewey et Bentley (1949), correspond pour nous à des interactions productives, le plus souvent associées à des rencontres (mais pouvant être également largement asynchrones), permettant la transformation d'un artefact médiateur et des personnes parties prenantes pour réaliser une performance ${ }^{2}$. Comme l'école de Montréal (Taylor \& Van Every, 2000), nous faisons référence à Greimas pour analyser le déroulement des transactions coopératives (par ex. Cooren, 1999) en réinterprétant toutefois un certain nombre de ses catégories ${ }^{3}$.

1 Nous désignons ces langages par l'expression de méta-sémiotique (voir, par exemple, Zacklad, 2010b).

2 Voir B. Simpson (2013) dans ce numéro, pour les différences entre les concepts d'interaction et de transaction.

3 L'exploitation de la sémiotique narrative de Greimas est également massive dans la théorie de l'acteur réseau sans que la reconnaissance de cette dette ait peut-être toujours été assez soulignée par les auteurs et les commentateurs. 
Par rapport à l'économie, la référence la plus proche elle celle de J. Commons (2001/1931) qui s'inscrit lui-même dans une perspective pragmatiste. Comme l'explique Delfavard dans Naves \& Defalvard (2006), alors que les économistes classiques valorisent la marchandise, point de vue des artefacts et que les économistes néo-classiques valorisent l'utilité, point de vue des sujets, l'unité d'analyse pour les institutionnalistes est celle de l'activité, c'est-à-dire de la transaction, de ses participants et des règles d'action collective dont ils se dotent. Néanmoins, chez Commons comme chez la plupart des institutionnalistes, le rôle médiateur des artefacts et de la technologie n'est pas pris en compte de manière satisfaisante.

Les personnes engagées dans la transaction sont en position de (co-)réalisateur, de (co)bénéficiaire, de (co-)destinateur (mandant) ${ }^{4}$ et de (co-)destinataire, toutes les configurations de symétrie et d'asymétrie entre ces positions étant possibles (cf. infra pour une définition). Une personne individuelle peut être engagée dans une transaction avec elle-même. Les réalisations de la transaction, aussi diverses qu'une conversation, un repas, le déplacement conjoint d'un objet matériel, constituent à la fois l'une de ses finalités et la condition nécessaire de la mise en relation des personnes. En effet, toute transaction doit être médiatisée, soit par les gestes et les paroles des personnes en présence, soit «à distance », par l'intermédiaire d'artefacts pérennes circulants d'un corps à l'autre (Zacklad, 2007a).

Une organisation peut être définie comme un ensemble de programmes transactionnels récurrent constituant des flux transactionnels obéissant à un ensemble de conventions et de règles dans un contexte donné (cf. infra). Les transactions opèrent toujours une transformation simultanée sur deux plans : celui des caractéristiques des artefacts médiateurs et celui du vécu expérientiel des personnes engagées dans la transaction (Figure 1). Parmi les artefacts médiateurs, on établit une distinction entre les artefacts porteurs de valeur et les artefacts capacitants. Selon notre terminologie, les artefacts sont aussi constitués par la gestualité des personnes quand celle-ci correspond à une structure reproductible affectant l'environnement y compris de manière transitoire comme dans la communication orale: vibrations sonores (parole), flux lumineux (langage corporel visible), sensations corporelles... Ces manifestations transitoires produites par le corps humain sont considérées comme des artefacts dès lors qu'elles constituent des signes cohérents, identifiables et reproductibles par les sujets.

\section{2.- Action, pratique, activité, transaction, démarche d'enquête, travail...}

Avant de poursuivre, nous proposerons quelques précisions terminologiques préliminaires. Action et pratique sont des notions que nous considérons comme désignant des niveaux de réalité équivalents. Le terme d'action est utilisé pour désigner des activités qui visent des finalités explicites, même si elles ne sont pas exprimées avec précision et dont les sujets sont en mesure de considérer, à l'issue d'un processus d'évaluation, qu'elles sont atteintes. L'action est inscrite dans une perspective diachronique qui possède un début et une fin et elle est associée à la réalisation d'une performance, au sens que nous donnerons plus bas à ce terme. La notion de transaction, ou transaction coopérative pour sortir de toute ambiguité avec l'acception économique classique du terme, renvoie pour nous à la théorie particulière de l'action que nous décrivons ici et qui met l'accent sur son caractère à la fois intrinsèquement social et instrumenté. En référence à Greimas, nous décrivons les transactions comme étant inscrites dans des programmes narratifs, des programmes transactionnels, qui contiennent quatre étapes: virtualisation, compétences, performance, évaluation (cf. infra).

Si l'on parle de pratique, en distinguant ce terme de celui d'action, comme dans les expressions de «pratiques professionnelles » ou de « communautés de pratique », on met davantage l'accent sur la compétence que sur la performance. La référence à la pratique fait référence à la maîtrise de savoir-faire qui sont indispensables à l'accomplissement de l'action, mais qui ne sont pas

4 Le terme de « destinateur » est utilisé par Greimas pour décrire l'actant à l'initiative du programme narratif que

l'on pourrait également appeler le « mandant ». 
envisagés sous l'angle de la performance réalisée. On parlera ainsi de pratiques d'évaluation, de pratiques liées à l'entretien de la compétence, ou de pratiques instrumentales spécifiques qui correspondent à des étapes du programme transactionnel. À ce titre, une pratique correspond à un épisode «libre » d'un programme transactionnel que l'on peut intégrer et recombiner dans d'autres programmes pour réaliser des actions liées à des performances.

L'action comme la pratique peuvent être inventives ou routinières (Tableau 1). Quand les transactions coopératives sont inventives, elles donnent lieu au déroulement d'une démarche d'enquête au sens donné à ce terme par Dewey (1938). Pour John Dewey, auteur central du pragmatisme à qui nous empruntons la conceptualisation de la transaction, l'enquête est une «forme fondamentale de l'activité humaine » (Gros, 2011) qui prend place dès que les sujets sont confrontés à une situation indéterminée, une situation de « discontinuité » entre le sujet en son environnement (Zacklad, 1993). Cette discontinuité amène les sujets à déclarer la situation «problématique » ou à instituer un problème. "L'enquête est la transformation contrôlée ou dirigée d'une situation indéterminée en une situation qui est si déterminée en ses distinctions et relations constitutives qu'elle convertit les éléments de la situation en un tout unifié. » (Dewey, 1938, p. 11). Dans un grand nombre de situations une fois le problème bien posé une solution apparait immédiatement. Mais la solution d'un problème n'est pas la solution d'une enquête, car celle-ci implique un «jugement» au sens judiciaire du terme, qui justifie les raisons pour lesquelles la solution peut être acceptée (Zacklad, 1993).

\begin{tabular}{|c|c|c|}
\hline & $\begin{array}{c}\text { Explicitement finalisée : action } \\
\text { (pouvant relever du travail) }\end{array}$ & Non explicitement finalisée : pratique \\
\hline Habituel & $\begin{array}{c}\text { Action habituelle }=\text { transaction relevant } \\
\text { d'une performance usuelle }\end{array}$ & $\begin{array}{c}\text { Pratique régulière = épisode d'un programme } \\
\text { transactionnel non assigné de manière univoque } \\
\text { à une performance }\end{array}$ \\
\hline Inventif (enquête) & $\begin{array}{c}\text { Action inventive associée à une } \\
\text { démarche d'enquête }=\text { transaction dont la } \\
\text { performance est inventive }\end{array}$ & $\begin{array}{c}\text { Pratique originale }=\text { épisode inventif non } \\
\text { assigné de manière univoque à une performance }\end{array}$ \\
\hline
\end{tabular}

Tableau 1 : Action et pratique selon l'inventivité et l'explicitation de la finalité

\section{Table 1: Action and practice according to the inventiveness and the explicitness of the goal}

Ainsi, si toute transaction est doublement productive sur le plan de la transformation des caractéristiques des artefacts médiateurs comme sur celui de la transformation de l'expérience des personnes, la nature de ces transformations diffère selon le caractère plus ou moins routinier de la transaction. Dans le cas des transactions routinières, nous considérons que les artefacts médiateurs ont le statut d'artefacts usuels constituant une réplique et que les personnes agissent en tant qu'agents. Dans le cas des transactions inventives, les artefacts médiateurs ont le statut d'œuvre, dans le sens donné par Meyerson (1948) à ce terme et les personnes agissent en tant que self autonome dans le sens de Mead (2006/1934) (Tableau 2).

\begin{tabular}{|c|c|c|}
\hline & $\begin{array}{c}\text { Plan de la transformation des caractéristiques } \\
\text { des artefacts médiateurs }\end{array}$ & $\begin{array}{c}\text { Plan de la transformation du vécu } \\
\text { expérientiel }\end{array}$ \\
\hline Transaction routinière & $\begin{array}{c}\text { Artefact usuel } \\
\text { (réplique) }\end{array}$ & Agent \\
\hline Transaction inventive & Cuvre & Self \\
\hline
\end{tabular}

Tableau 2 : Statut des artefacts et des personnes selon le caractère routinier ou inventif de la transaction

Table 2: Status of artifacts and people according to the routine versus inventive nature of the transaction

Dans l'action comme dans la pratique, on parlera d'activité quand la perspective adoptée est celle des sujets et de processus techniques quand la perspective adoptée est celle des artefacts médiateurs. L'analyse d'une pratique d'écriture, par exemple, pourra ainsi se faire d'une 
manière symétrique du point de vue des activités rédactionnelles (perspective du sujet), gestualité, représentations des contenus, intentions de communication pragmatiques ${ }^{5}$, comme du point de vue des processus techniques d'élaboration progressif du texte (point de vue de l'artefact) en suivant simultanément les transformations des propriétés tangibles du support, les caractéristiques expressives de la langue utilisée et celles du texte en cours d'élaboration et les principes rhétoriques permettant d'orienter la relation avec les lecteurs.

La notion de travail correspond pour nous à des actions instituées par la reconnaissance de leur valeur sociale, c'est-à-dire considérées comme conformes aux intérêts d'une communauté. Le travail inclut de très nombreuses pratiques. Selon les contextes, les sociétés, les systèmes politiques, différents critères pourront intervenir pour conférer à des actions une reconnaissance en tant que travail. La formalisation d'une relation entre un destinateur ${ }^{6}$ (ou mandant), à l'origine du projet transactionnel et un destinataire, qui l'évalue, est pour nous un des critères majeurs. Rappelons que dans l'interprétation que nous faisons du modèle actantiel de Greimas (1966) destinateur et destinataire sont des actants différents du réalisateur et du bénéficiaire même si ces rôles peuvent être assumés par la même personne (cf. infra).

\section{3.- Situation vs Contexte}

En suivant Joelle Zask (2008) nous introduisons la distinction entre situation et contexte qui permet de dépasser certaines limitations du dialogue parfois stérile entre approches interactionnistes et approches structuralistes du social qui opposent la créativité et la subjectivité de l'agir en situation à la normativité et à l'objectivation des structures sociales déterminant l'action (statuts, positions, rôles prédéfinis...). Les mêmes oppositions se retrouvent dans certains usages des catégories ergonomiques de travail réel versus travail prescrit sans que l'enrichissement réciproque entre ces deux dimensions ne soit toujours pris en compte ${ }^{7}$.

5 On ne confondra pas «pragmatique » au sens linguistique de dimension d'analyse de la communication et «pragmatiste » au sens du courant philosophique essentiellement nord-américain auquel nous nous référons (on pourra parler aussi, pour désambiguïser de philosophie pragmatique).

6 «Destinateur » est le terme utilisé par Greimas pour désigner l'initiateur du programme narratif. Il correspond à celui de mandant qui fait appel à un mandataire, le réalisateur (cf. Figure 1).

7 Différents auteurs en ergonomie ou en sociologie du travail ont tenté de dépasser cette opposition soit en développant la notion de genre professionnel comme un intermédiaire entre le réel et le prescrit (Clot \& Faïta, 2000) soit en adressant explicitement la notion de travail d'organisation (de Terssac \& Maggi, 1996). Tout en souscrivant à la pertinence de ses approches nous remarquons qu'elles adressent essentiellement la question sous l'angle de l'auto-organisation, de la création autonome, ascendante des prescriptions, un aspect essentiel mais qui n'intègre pas toutes les dimensions du management. 


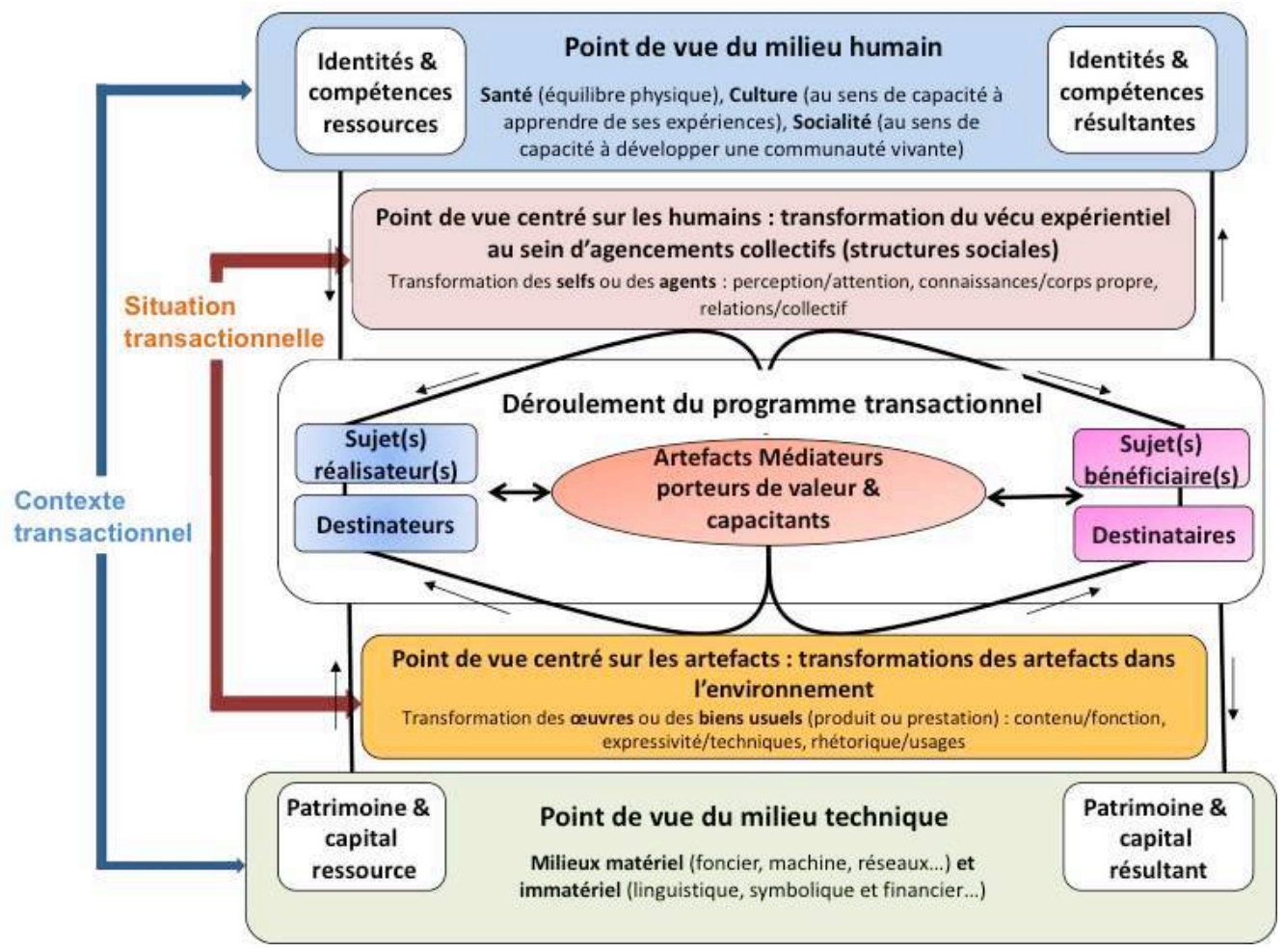

Figure 1 : Schématisation de la structure des transactions coopératives

\section{Figure 1: Diagram of the structure of cooperative transactions}

J. Zask commence par rappeler l'inspiration darwienne de la philosophie de John Dewey «en développant ses pouvoirs, un organisme influe sur le milieu de sa vie et réciproquement le milieu conditionne ceux de ses pouvoirs susceptibles d'un développement »(pp. 313-314). En suivant la philosophie de cet auteur elle propose de nommer situation, «tous les moments au cours desquels l'interaction entre un vivant et un milieu s'effectue sous la forme d'une action réciproque » et contexte les «moments menant à la conformation passive du premier aux conditions $d u$ second» (p. 314). Au plan organique cette distinction recouvre la distinction entre l'environnement (situation) et le milieu (contexte).

Par ailleurs, dans l'épistémologie anti-dualiste du pragmatisme (Steiner, 2008) la distinction entre environnement et organisme doit être comprise «comme des phases de différenciation, non comme des essences permanentes ou des êtres en soi $\gg$. Cette approche trouve un écho naturel dans les approches instrumentales : l'outil relève de l'environnement et nécessite une adaptation du sujet pour que celui-ci se l'approprie, l'instrument est intégré à des schèmes d'action, il est du côté du sujet et permet à celui-ci d'agir sur l'environnement (Rabardel, 2005). Il n'y a donc pas de distinction ontologique entre outils et instruments qui relèvent de degré d'apprentissages différents. Ceci est d'autant plus patent pour les «artefacts gestuels» comme les habiletés entièrement incorporées au sujet, parler une langue étrangère, par exemple. La compétence linguistique peut être considérée comme intégrée au sujet, ou la langue et ses règles peuvent être considérées comme faisant partie d'un milieu symbolique et culturel externe que le sujet doit s'approprier. Selon la terminologie piagétienne, on peut considérer que dans la phase d'accommodation l'environnement est d'abord disjoint du sujet avant d'être progressivement intériorisé par l'assimilation qui permettra un couplage fonctionnel.

La séparation entre le sujet et les artefacts médiateurs qui lui servent d'instrument et qu'il coproduit durant les transactions est donc une question de perspective. S'il y a bien toujours un 
organisme individuel ou collectif qui relève de l'ordre du vivant et qui est le sujet de l'action ${ }^{8}$, l'incorporation au sujet de certains artefacts instrumentaux dans la description d'une situation transactionnelle, ou leur attribution à l'environnement, ses propriétés et ses ressources, dépend de la perspective choisie et des finalités de l'étude. Par ailleurs, l'environnement varie selon les espèces et leurs activités "L'environnement d'un poisson est distinct de celui d'une abeille. » Enfin, "plus la forme de vie est élevée et complexe, plus les activités de reconstruction du milieu sont intenses et variées, plus le degré auquel les vivants influent sur leur environnement au cours de leur quête de ressources propres est important. »(Zask, 2008, p. 314).

À l'inverse de la situation, le «contexte est un milieu dans lequel prend place telle ou telle conduite: un discours, une action, une croyance, etc. S'il détermine les significations et les traits de cette conduite, il n'est pas en retour affecté par elle. Les caractéristiques d'un contexte sont pensées comme indépendantes des conduites que l'on y réfère. Elles sont antérieures et peuvent être connues par elles-mêmes, indépendamment des conduites particulières qui en semblent alors des variations accidentelles.»(Ibid.). Dans son texte, J. Zask met surtout l'accent sur le caractère limitant du contexte, celui-ci restreignant le possible, limitant l'action. Mais le contexte fournit également des ressources qui sont intégrées à la situation et permettent son développement. Dans une situation transactionnelle, "une enquête conclusive signifie une enquête procurant un matériau susceptible d'être à son tour converti en des moyens pour d'autres fins, donc pour des expériences ultérieures » (Ibid. p. 317).

Pour la sémiotique des transactions coopératives, ces artefacts porteurs de valeur issus d'une situation transactionnelle sont susceptibles de se transformer en artefacts capacitants pour d'autres transactions (cf. infra) et deviennent les constituants d'un contexte pour une communauté de pratique ou d'action. À ce titre, ils sont susceptibles de constituer du «commun» au sens où les économistes utilisent ce terme (cf. par exemple, Ostrom 1990 ; Polanyi 1983).

\section{4.- L'artefact médiateur en tant que finalité ou moyen}

Une distinction majeure entre les artefacts est celle qui sépare l'artefact conçu en tant que finalité de l'activité, artefact porteur de valeur et les artefacts conçus en tant que moyens permettant d'atteindre cette finalité, artefacts capacitants. Un objet de consommation est un artefact en tant que finalité. Un outil est un artefact capacitant destiné à amplifier les aptitudes de son utilisateur dans une situation donnée. Cette distinction peut être croisée avec celle de la prise en considération du destinataire principal de l'artefact, soi-même ou un tiers (Tableau 3). Comme on le verra, cette distinction ne repose pas sur des caractéristiques «intrinsèques » des artefacts, mais dépend de leur statut dans le programme transactionnel.

\begin{tabular}{|c|l|l|}
\hline & \multicolumn{1}{|c|}{ Pour soi } & \multicolumn{1}{c|}{ Pour un tiers } \\
\hline $\begin{array}{c}\text { En tant que finalité (artefact } \\
\text { porteur de valeur) }\end{array}$ & $\begin{array}{l}\text { Objet de consommation personnel } \\
\text { (pomme de terre cuite pour la } \\
\text { consommation directe) }\end{array}$ & $\begin{array}{l}\text { Objet destiné à l'échange, produit } \\
\text { (pomme de terre sur l'étalage d'un } \\
\text { marchand) }\end{array}$ \\
\hline $\begin{array}{c}\text { En tant que moyen } \\
\text { (artefact capacitant) }\end{array}$ & $\begin{array}{l}\text { Instrument ou technique personnel } \\
\text { (pomme de terre ingrédient pour faire } \\
\text { un gratin) }\end{array}$ & $\begin{array}{l}\text { Technique, machine de « production » } \\
\text { (pomme de terre en tant que semence) }\end{array}$ \\
\hline
\end{tabular}

Tableau 3 : Les artefacts en tant que finalité ou moyen, pour soi ou pour un tiers

Table 3: Artifacts as purpose or means, for oneself or for a third-party

Par exemple, une pomme de terre considérée comme un légume à consommer, sera un artefact

8 Au moins dans la tradition la tradition non animiste et non totémiste de nos sciences humaines et sociales pour les quelles seuls les humains, ou certains animaux, sont dotés d'intériorité (cf. infra la référence à Descola, 2002, 2005). 
médiateur en tant que finalité ou artefact porteur de valeur, un objet de consommation destinée à soi-même ou à partager. Le même légume, sur l'étalage d'un marchand et du point de vue de celui-ci sera un artefact porteur de valeur destiné à un tiers, un produit. Toujours dans la sphère domestique, pour la cuisinière préparant un gratin, la pomme de terre sera un instrument nécessaire à la réalisation d'un plat, un artefact capacitant périssable (capital circulant ou périssable), devant à la fois être composé avec d'autres et faire l'objet de traitement (épluchage, coupe, etc.). Enfin, la pomme de terre pourrait être considérée comme "semence » destinée à faire l'objet d'une mise en culture chez un tiers où elle deviendra le composant d'un procès de production (artefact en tant que moyen pour un tiers). La même analyse pourrait être conduite pour un artefact sémiotique, un discours dans le cadre d'un cours, qui pourrait être considéré comme un produit pour les étudiants et comme un instrument pour l'enseignant utilisant cette opportunité pour clarifier sa pensée.

\section{5.- Sujets et artefacts selon la physicalité et l'intériorité}

Les sciences cognitives contemporaines concernées par l'étude de l'activité, comme le courant de cognition située (Suchman, 1987) ou de la cognition distribuée (Hutchins, 1995) que l'on peut considérer comme étant inscrites dans le tournant pragmatique (Steiner 2008) réfutent fermement l'idée selon laquelle les comportements manifestes seraient causalement déterminés par des processus intracrâniens se déroulant de manière solipsiste (Brassac, 2004). Mais aucun compte rendu de l'activité n'échappe au fait que celle-ci est tantôt décrite du point de vue de ses manifestations comportementales (emplacement des corps, déplacements, transformations matérielles des artefacts...), tantôt décrite du point de vue des intentions des sujets renvoyant à leur intériorité (intention, intérêt, stratégie, etc.). Cette dualité de point de vue dans la narration de l'action semble assez universelle. L'anthropologue Philippe Descola $(2002,2005)$ utilise ainsi les dimensions de la physicalité et de l'intériorité pour pointer les divisions entre les êtres qu'elle autorise en identifiant quatre grandes ontologies, (1) naturaliste, celle des sciences occidentales, (2) totémique, (3) animiste et (4) analogique.

«Par intériorité, il faut certes entendre la gamme des propriétés ordinairement associées à l'esprit, à l'âme ou à la conscience - intentionnalité, subjectivité, réflexivité, affects, aptitude à signifier ou à rêver - , mais aussi les principes immatériels supposés causer l'animation, tels le souffle ou l'énergie vitale, en même temps que des notions plus abstraites comme l'idée que je partage avec autrui une même essence, un même principe d'action ou une même origine. Par contraste, la physicalité concerne la forme extérieure, la substance, les processus physiologiques, perceptifs et sensori-moteurs, voire le tempérament ou la façon d'agir dans le monde en tant qu'ils manifesteraient l'influence exercée sur les conduites ou les habitus par des humeurs corporelles, des régimes alimentaires, des traits anatomiques ou un mode de reproduction particuliers. » (p. 1) (Descola, 2005)

L'approche naturaliste de la science occidentale dénie ainsi aux non-humains toute forme d'intériorité qui pourrait être assimilable à celle des humains, comme le fait de posséder des «intentions ». Pour effectuer des descriptions qui symétrisent le rôle des humains et des nonhumain $\mathrm{s}$ ou qui neutralisent pour un temps les explications faisant appel à l'intériorité, les tenants de la cognition distribuée ou de la théorie de l'acteur-réseau (Akrich, Callon, \& Latour, 2006) s'en tiendront donc à des descriptions essentiellement physicaliste de la pratique (cf. Tableau 4). Sans considérer que les processus relevant de l'intériorité seraient des déterminants causaux exclusifs des mouvements physiques attribués aux corps et sans bien sur adopter non plus la position inverse, la sémiotique des transactions coopératives identifie ainsi deux genres de description parallèle de l'action privilégiant le point de vue de l'intériorité versus celui de l'externalité ou physicalité (Tableau 4). 


\begin{tabular}{|l|l|l|}
\hline En intériorité & Personnes & Artefact \\
\hline $\begin{array}{l}\text { En physicalité (ou } \\
\text { externalité) }\end{array}$ & $\begin{array}{l}\text { Esprit : Valeurs, désir, } \\
\text { connaissances, perception, } \\
\text { représentations, sentiments, } \\
\text { relations... }\end{array}$ & $\begin{array}{l}\text { Dominante sémiologique : valeur symbolique, } \\
\text { contenu, signification, fonction d'usage, esthétique, } \\
\text { médiation symbolique... }\end{array}$ \\
$\begin{array}{l}\text { Corps : Pulsion, attention, } \\
\text { mouvements, automatismes, } \\
\text { imitation, mouvements de foule... }\end{array}$ & $\begin{array}{l}\text { Dominante tangible : données objectivées faisant } \\
\text { matérielle, fonction technique, formes physiques, } \\
\text { médiation physique... }\end{array}$ \\
\hline
\end{tabular}

Tableau 4 : Les personnes et les artefacts en intériorité et en physicalité

Table 4: People and artifacts in interiority and physicality

\section{3.- Orientation ternaire de l'activité}

La STC considère que l'activité est orientée selon trois directions complémentaires, récurrentes et entrelacées, à la fois dans le cadre des activités d'enquêtes et dans celui des activités routinières ou habituelles, approche ternaire qui fait écho à la stratification de l'action en trois couches chez A. Giddens (1984), motivation, rationalisation, contrôle réflexif, même si cet auteur reste à un niveau plus macrosociologique dans ses conceptualisations. Dans les activités routinières, ces activités correspondent à trois formes d'orientation de l'attention des sujets, orientation temporelle, orientation normative et en justification et orientation réflexive qui interviennent dans le pilotage de l'action. Dans le cadre des transactions inventives, qui impliquent une la redéfinition du contexte transactionnel et la mise en place d'une enquête, ces trois orientations correspondent à des modalités de cadrage de l'action : gestion temporelle et motivationnelle du déroulement de l'enquête, registres de rationalisation, registres de contrôle de la cohérence de son déroulement (Figure 2).

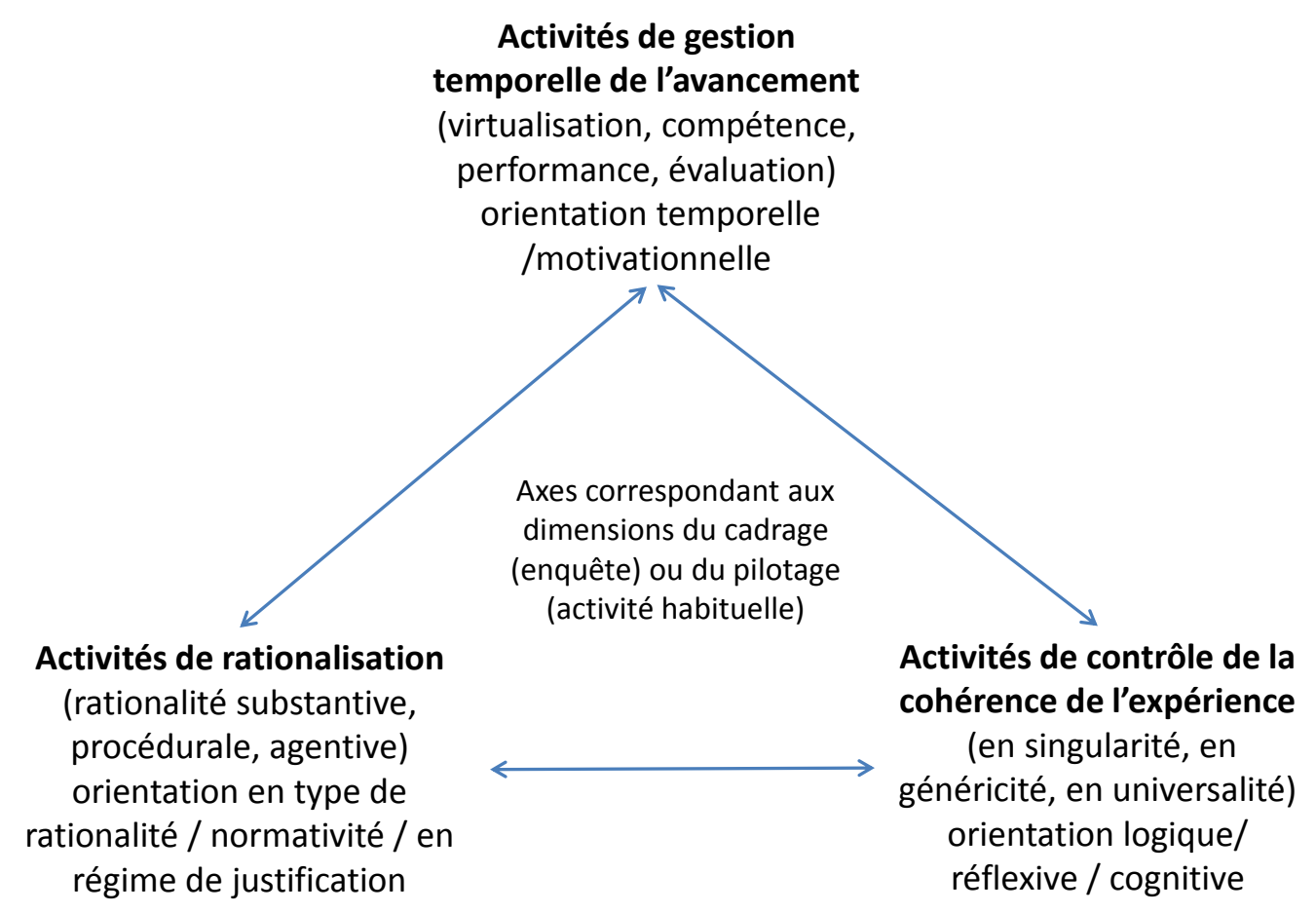

Figure 2 : Trois axes d'analyse logico-sémiotique de l'activité selon l'approche transactionnelle

Figure 2: Three axes of the logico-semiotic analysis of activity according the transactional approach 
Ces dimensions sont décrites à l'aide de la sémiotique narrative de Greimas et de la logique pragmatiste, celle de Dewey dans sa théorie de l'enquête et celle de Pierce qui est également une sémiotique ${ }^{9}$. Le recours à la sémiotique est en ligne avec les approches culturalistes de la cognition et de l'action qui sont elles-mêmes au cœur du pragmatisme, qu'il s'agisse de l'inscription sociale de la conduite humaine chez G. H. Mead (Brassac, 2007) ou bien sur de l'œuvre de l'œuvre de C. S. Peirce, un des fondateurs de cette discipline, pour qui le raisonnement logique est isomorphe aux processus d'enquêtes instrumentées des sujets. Cette approche culturaliste suggère d'ailleurs des rapprochements originaux avec le courant de la psychologie de l'activité inspirés par L. S. Vygotsky (Brassac, 2007). La STC recourt aussi aux approches narratives qui sont centrales dans la vision culturaliste de la cognition telles que promues, par exemple, par J. Bruner («The Narrative Construction of Reality », 1991) et dont une partie de l'inspiration remonte aux travaux du linguiste V. Propp $(1928,1970)$ qui ont également largement inspiré Greimas.

Selon ces approches, les notions mobilisées par les scientifiques pour analyser l'action ne sont pas intrinsèquement différentes de celles mobilisées par les sujets eux-mêmes pour rendre compte de la cohérence de leurs actions. Elles sont simplement davantage contrôlées et systématisées, ce qui entraîne la construction d'une terminologie spécialisée et de méthodes de comptes rendus rigoureuses visant, autant que possible, à éviter les ambiguïtés et certains pièges du sens commun. Si la réalité est construite de manière narrative, pour reprendre le terme de $\mathrm{J}$. Bruner (1991), elle l'est à la fois pour le sens commun et pour la science et une des tâches des analystes de l'activité est dégager les principes sémiotiques systématisés permettant de mettre en relief tels ou tels aspects de l'activité et des comportements. Cette systématisation vise à faciliter l'opérationnalisation dans les situations empiriques et à développer les connaissances dans les différents domaines des sciences humaines et sociales: psychologie, économie, sociologie, gestion, anthropologie, sciences de la communication, etc.

Il nous semble d'ailleurs que de manière plus ou moins explicite, tous les chercheurs en sciences humaines et sociales se dotent, pour instrumentaliser leur théorie, d'une métasémiotique plus ou moins rigoureuse leur permettant de rendre compte du comportement des acteurs, de leur motivation, de leurs représentations, de leurs instruments, de leurs modalités d'interaction avec leurs pairs comme avec l'environnement. Les descriptions scientifiques sont à la fois inspirées des rationalisations spontanées des sujets et contribuent ensuite à les façonner du fait du caractère performatif des résultats des sciences humaines et sociales (Muniesa \& Callon, 2009).

\section{1.- Activités de gestion temporelle du déroulement de l'activité et d'entretien de la motivation}

Dans cette partie nous présenterons les activités de gestion temporelle des démarches d'enquête qui peuvent également être appréhendées sous l'angle de l'entretien de la motivation des sujets, pour reprendre l'expression de Giddens. Nous introduirons également les notions de sémiotique narrative qui nous seront utiles pour analyser l'activité selon les autres perspectives. La sémiotique narrative de Greimas (1966), interprétée par Everaert-Desmedt (1990) et Hebert (2006) a déjà été exploitée dans le domaine de la communication organisationnelle par Coreen (1999). Précisons d'emblée que la référence aux théories narratives ne signifie nullement que nous considérons que l'action et la pratique ne sont constituées que de récits qu'il suffirait d'organiser. Nous utilisons la sémiotique narrative comme un cadre logique en mesure de rendre compte de toute «action réelle ou thématisée » (Hebert, 2006), thématisée signifiant mise en récit. Les catégories de la méta-sémiotique greimassienne ont pour nous le même statut que les catégories de sujet, instrument et objet dans la théorie de l'activité (par exemple, Engeström, 1987) : elles offrent un cadre analytique pour rendre compte de l'action finalisée, les

9 D'autres analystes de l'activité, tel Jacques Theureau (2004) utilisent également la sémiotique pragmatiste pour rendre compte du cours d'action et des comparaisons restent à faire entre ces approches qui excèdent la place qui nous est impartie. 
textes littéraires n'étant qu'une forme de description parmi d'autres de ces actions.

La méta-sémiotique de Greimas a connue deux versions, le modèle actanciel, puis le schéma narratif canonique, qui définissaient six puis quatre actants permettant de rendre compte de la logique des récits. Nous nous inspirerons librement de ces catégories et de certains concepts en fonction de notre objectif de description d'activités essentiellement productives et coopératives, même si la coopération n'exclut pas le conflit. Voici une description des six actants du modèle actanciel de Greimas proposée par Hebert (2006) :

«Axe du vouloir (désir) : (1) sujet / (2) objet. Le sujet est ce qui est orienté vers un objet. La relation établie entre le sujet et l'objet s'appelle jonction. Selon que l'objet est conjoint au sujet (par exemple, le prince veut la princesse) ou lui est disjoint (par exemple, un meurtrier réussit à se débarrasser du corps de sa victime), on parlera, respectivement, de conjonction et de disjonction.

Axe du pouvoir: (3) adjuvant / (4) opposant. L'adjuvant aide à la réalisation de la jonction souhaitée entre le sujet et l'objet, l'opposant y nuit (par exemple, l'épée, le cheval, le courage, le sage aident le prince ; la sorcière, le dragon, le château lointain, la peur lui nuisent).

Axe de la transmission (axe du savoir, selon Greimas) : (5) destinateur / (6) destinataire. Le destinateur est ce qui demande que la jonction entre le sujet et l'objet soit établie (par exemple, le roi demande au prince de sauver la princesse). Le destinataire est ce pour qui la quête est réalisée. En simplifiant, interprétons le destinataire (ou destinataire-bénéficiaire) comme ce qui bénéficiera de la réalisation de la jonction entre le sujet et l'objet (par exemple, le roi, le royaume, la princesse, le prince, etc.). Les éléments destinateurs se retrouvent souvent aussi destinataires. » (www.signosemio.com/greimas/modele-actantiel.asp, consulté le 11/09/2011).

Dans l'état actuel de notre réflexion, nous proposons de nous appuyer sur six actants permettant de définir le programme transactionnel : (1) le destinataire et (2) le destinateur, (3) le sujet réalisateur et le (4) sujet bénéficiaire, (5) l'artéfact médiateur porteur de valeur (ou objet porteur de valeur) et les (6) artefacts médiateurs capacitants (Figure 3). D'autres sous-catégories secondaires seront mobilisées dans l'analyse: les sujets et les destinataires pourront être analysés en tant que self ou agent, les artefacts médiateurs en tant qu'objet usuel ou œuvre, ils pourront être, comme nous l'avons vu, externes ou incorporés et les artefacts capacitants pourront être virtualisant, qualifiant ou évaluatif, selon l'épisode de la transaction où ils interviennent. Les rôles d'opposant, les anti-sujets, ne sont pas décrits à ce stade.

\begin{tabular}{|c|c|c|c|c|}
\hline $\begin{array}{c}\text { Destinateur } \\
\text { (ou mandant) }\end{array}$ & $\begin{array}{c}\text { Artefact } \\
\text { médiateur } \\
\text { évaluatif } \\
\text { (expérientiel) }\end{array}$ & $\begin{array}{c}\text { Artefact porteur de } \\
\text { valeur secondaire } \\
\text { (gratifiant) }\end{array}$ & $\begin{array}{c}\text { Artefact } \\
\text { médiateur } \\
\text { évaluatif } \\
\text { (instrumental) }\end{array}$ & Destinataire \\
\hline $\begin{array}{c}\text { Artefact } \\
\text { médiateur } \\
\text { virtualisant } \\
\text { (ex-ante) }\end{array}$ & 1 & 3 & $\begin{array}{c}\text { Artefact } \\
\text { médiateur } \\
\text { virtualisant } \\
\text { (ex-post) }\end{array}$ \\
\hline $\begin{array}{c}\text { Sujet } \\
\text { réalisateur }\end{array}$ & $\begin{array}{c}\text { Artefact } \\
\text { médiateur } \\
\text { qualifiant } \\
\text { (instrumental) }\end{array}$ & $\begin{array}{c}\text { Artefact porteur de } \\
\text { valeur principal } \\
\text { (consommatoire) }\end{array}$ & $\begin{array}{c}\text { Artefact } \\
\text { médiateur } \\
\text { qualifiant } \\
\text { (expérientiel) }\end{array}$ & $\begin{array}{c}\text { Sujet } \\
\text { bénéficiaire }\end{array}$ \\
\hline
\end{tabular}

Figure 3 : Une représentation en tableau des actants du programme transactionnel : l'artefact porteur de valeur secondaire peut correspondre à un remerciement ou à une rétribution monétaire

Figure 3: Representation in table form of the actants of the transactional program: the secondary value-carrying artifact may correspond to gratitude or to a monetary reward 
Nous introduisons l'actant «sujet bénéficiaire» en le distinguant du destinataire. Les destinataires évaluent l'artefact porteur de valeur sans être nécessairement les bénéficiaires directs de sa mise en œuvre, au sens d'une forme de consommation ou d'expérience. Néanmoins, leurs évaluations sont souvent basées sur la prise en considération de l'expérience que le bénéficiaire direct fait de l'artefact porteur de valeur. Dans de nombreuses situations économiques ou organisationnelles cette différenciation est présente: le destinateur est l'entrepreneur qui initie un projet avec l'aide de financeurs, les destinataires sont les financeurs et l'entrepreneur lui-même, les sujets réalisateurs sont les salariés et les sujets bénéficiaires les clients (cf. Figure 2).

Selon le principe du «syncrétisme actanciel », le même élément peut occuper plusieurs classes actantielles. Par exemple, dans une transaction qui n'impliquerait qu'une seule personne, celleci peut simultanément occuper toutes les positions de destinateur, sujet réalisateur, sujet bénéficiaire puis destinataire. Elle sera destinatrice en formulant le projet de préparer un repas pour répondre à une faim soudaine, un objet de valeur. Elle sera ensuite sujet réalisateur dotée d'artefacts capacitants externes et internes, livre de recettes de cuisine et savoir-faire liés à la préparation. Elle sera sujet bénéficiaire en dégustant sa préparation puis destinataire en estimant que sa faim a été comblée en tenant compte de divers critères : goût, diététique, etc.

Dans une action coopérative à plusieurs, ces rôles peuvent être occupés par des personnes différentes. Le destinateur (mandant) peut être la mère de l'enfant, celui-ci étant le sujet bénéficiaire. Le grand-père peut être le sujet réalisateur préparant le plat à la demande de la mère et le père, la mère et l'enfant trois destinataires évaluant positivement le repas après consommation. Dans cette histoire, il peut exister d'autres artefacts porteurs de valeurs non perceptibles par un tiers au premier abord. Par exemple, la contribution du grand-père à la préparation du repas peut-être un artefact porteur de valeur en soi, un comportement coopératif dont la bénéficiaire principale est la mère. Dans les situations réelles, les programmes transactionnels sont enchâssés de multiples manières, s'interrompent, reprennent, se complètent, s'hybrident. Dans toute situation économique, il y a simultanéité de plusieurs flux transactionnels impliquant de multiples actants, présents et absents. Imaginons l'exemple du repas dans un contexte de restauration où interviennent un responsable de la promotion du restaurant, un propriétaire-patron, un cuisinier, des serveurs, des clients invités par d'autres clients payeurs, etc.

En nous inspirant du schéma narratif canonique de Greimas (Figure 4), on identifie quatre étapes ou épisodes dans le programme transactionnel: (1) la virtualisation ${ }^{10}$ (le destinateur exprime la vision du projet qui sera réalisé par le sujet réalisateur), (2) l'acquisition de compétences (les sujets réalisateurs mobilisent ou acquièrent les artefacts capacitants), (3) la performance (la production, coproduction, acquisition, consommation ${ }^{11}$ de l'artefact porteur de valeur impliquant les bénéficiaires), (4) l'évaluation ${ }^{12}$ (gratification, reconnaissance ou au contraire sanction négative exprimée par le destinataire au sujet réalisateur et/ou au destinateur). L'analyse narrative des transactions nous permet d'affiner les perspectives sur l'artefact médiateur en tant que finalité ou en tant que moyen. Dans la sémiotique narrative de Greimas, les programmes narratifs ont pour finalité le transfert d'un « objet de valeur », correspondant à un artefact médiateur appréhendé en tant que finalité. Dans le cadre de la STC, qui vise en particulier à rendre compte d'activités productives coopératives en particulier dans le secteur des services, nous considérons que la transaction correspond souvent à la coproduction d'un

10 Correspondant à l'étape de «manipulation » dans le schéma narratif de Greimas. La virtualisation renvoie à la futurité évoqué par Renault (dans ce numéro).

11 Dans les activités de production de service, qui occupent un rôle essentiel dans l'économie, la co-production et la consommation sont souvent simultanées. Il en est de même dans un grand nombre de pratiques non économiques : participer à des conversations, jouer à des sports, etc.

12 «anction » chez Greimas. 
artefact porteur de valeur constituant l'aboutissement du projet transactionnel individuel ou collectif. Dans les transactions coopératives, l'artefact porteur de valeur est plus souvent produit ou créée que transféré.

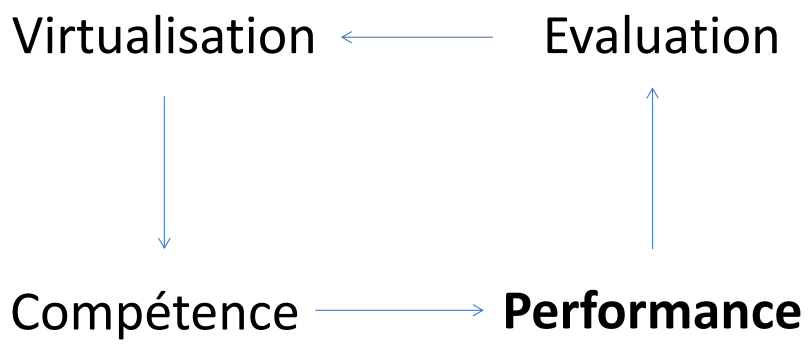

Figure 4 : Les quatre épisodes canoniques des programmes transactionnels

Figure 4: The four canonical episodes of transactional programs

Pour différencier ces artefacts de l'artefact porteur de valeur coproduit dans l'étape de performance et pour insister sur le fait qu'ils visent à mettre le sujet en « capacité » de conduire ou de conclure la transaction, nous parlerons à leur sujet d'artefacts capacitants (Tableau 5). Chaque épisode de la transaction coopérative est caractérisé par la mobilisation ou la (co)production d'artefacts capacitants spécifiques qui tirent leur nom de l'épisode dont ils sont issus. Les personnes sont également transformées durant ces différents épisodes, ils acquièrent la vision d'un projet, acquièrent des compétences, font l'expérience de la consommation ou de l'usage d'un artefact porteur de valeur, etc. (Tableau 5) :

- Virtualisation : artefacts virtualisants et production d'expériences entretenant la motivation et l'engagement dans le projet - le plus souvent des artefacts sémiotiques produits par le destinateur et visant à engager le sujet réalisateur dans le programme transactionnel ;

- Compétence : artefacts qualifiants et production d'expériences développant la compétence des sujets - les artefacts externes et/ou incorporés (outil, documents, connaissances, savoirfaire, schèmes d'action instrumentaux...) qui constituent des moyens nécessaires à la réalisation de la performance par le sujet réalisateur ;

- Performance : artefacts porteurs de valeur qui sont transférés du réalisateur au bénéficiaire ou coproduits par ces acteurs et expériences associées à ces échanges - dans cette étape, les relations entre réalisateurs et bénéficiaires sont au centre du processus, que l'on privilégie le transfert, la consommation, ou la co-production des artefacts médiateurs considérés comme externes aux sujets ou la transformation expérientielle, pouvant être symétrique, des sujets impliqués dans la transaction ;

- Évaluation : artefacts externes et/ou incorporés (document, logiciels, savoir-faire, éléments symboliques, monnaie...) permettant soit (1) d'évaluer la performance soit (2) de fournir des gratifications ou des éléments de reconnaissances distincts de l'artefact porteur de valeur au sujet réalisateur et/ou au destinateur (en changeant de perspective, certains artefacts évaluatifs peuvent constituer des artefacts porteur de valeur dans des transactions symétriques décalées $\left.{ }^{13}\right)$.

13 Par exemple, dans une relation marchande qui serait constitué par des éléments de performances répétés et disjoints, la contrepartie monétaire peut constituer simultanément un artefact porteur de valeur pour le sujet réalisateur et un artefact évaluatif pour le bénéficiaire (rémunération d'un artisan). Dans une vente, où l'échange est synchrone et instantané, l'échange d'un bien matériel contre une somme d'argent correspond à la circulation 


\begin{tabular}{|l|l|l|l|l|}
\hline & $\begin{array}{l}\text { Virtualisation } \\
\text { (capacitation ex- } \\
\text { ante) }\end{array}$ & $\begin{array}{l}\text { Compétence } \\
\text { (capacitation ex-ante) }\end{array}$ & $\begin{array}{l}\text { Performance } \\
\text { (création de valeur) }\end{array}$ & $\begin{array}{l}\text { Évaluation } \\
\text { (capacitation ex-post) }\end{array}$ \\
\hline Étapes & $\begin{array}{l}\text { Élaboration ou co- } \\
\text { élaboration du projet } \\
\text { transactionnel }\end{array}$ & $\begin{array}{l}\text { Acquisition par les } \\
\text { réalisateurs ou les } \\
\text { coréalisateurs des } \\
\text { savoirs, instruments, } \\
\text { ressources }\end{array}$ & $\begin{array}{l}\text { Production, co- } \\
\text { production, acquisition, } \\
\text { consommation d'un } \\
\text { artefact porteur de } \\
\text { valeur }\end{array}$ & $\begin{array}{l}\text { Gratification, } \\
\text { reconnaissance de la } \\
\text { performance du réalisateur } \\
\text { par le bénéficiaire }\end{array}$ \\
\hline Artefacts & $\begin{array}{l}\text { Artefact capacitant } \\
\text { virtualisant }\end{array}$ & $\begin{array}{l}\text { Artefact capacitant } \\
\text { qualifiant }\end{array}$ & $\begin{array}{l}\text { Artefact porteur de } \\
\text { valeur }\end{array}$ & $\begin{array}{l}\text { Artefact capacitant } \\
\text { évaluatif }\end{array}$ \\
\hline
\end{tabular}

Tableau 5 : Étapes des transactions coopératives et artefacts médiateurs mobilisés et/ou (co)produits

Table 5: Stages of cooperative transactions and mediated artifacts mobilized and/or (co)produced

\section{2.- Activités de rationalisation des transactions}

Pour rendre compte des activités de rationalisation et les relations actantielles qui permettent de problématiser la transaction, nous nous inspirons d'un autre auteur majeur du pragmatisme, C.S. Pierce (2002), dont la théorie de la signification exploite trois catégories logiques qui structurent le processus sémiotique: la priméité, la secondéité et la tiercéité. De façon originale et espérons-le pas trop déroutante pour les spécialistes de ces auteurs, mous mettrons ces catégories en relation avec la sémiotique narrative de Greimas pour en déduire une typologie des modalités de rationalisation s'appliquant aux sujets comme aux artefacts médiateurs. Rappelons les catégories logiques proposées par Pierce selon Everaert-Desmedt (1990) qui permettent de décrire le parcours sémiotique suscité par un signe simple ou complexe.

- La priméité correspond à la «conception de l'être indépendamment de toute autre chose » (Everaert-Desmedt 1990), c'est catégorie de la qualité, du sentiment, des émotions, une pure potentialité dans le registre du possible ;

- La secondéité est la «conception de l'être relatif à quelque chose d'autre (ibid. p. 35), c'est la catégorie du réel, de l'expérience du fait d'existence, d'une chose, d'un évènement, d'une idée, d'une situation...;

- La tiercéité «est la médiation par quoi un premier et un second sont mis en relation» (pp.35-36), c'est la catégorie de la règle, de la loi qui se manifeste à travers des faits (secondéité) qui actualisent des qualités.

En exploitant de manière libre ces catégories, nous proposons de considérer que chacune d'elle correspond à des formes de rationalité éclairant de manière complémentaire la situation transactionnelle (Figure 5) :

- Rationalité substantive ${ }^{14}$ et orientation de l'activité selon l'axe du vouloir-faire ou du désir : l'action est justifiée par les caractéristiques du résultat, de l'artefact porteur de valeur, sur la base d'une stricte séparation entre fins et moyen sans prise en compte de la manière dont le processus de réalisation peut conditionner lui-même la définition de la performance.

- Rationalité procédurale et orientation de l'activité selon l'axe du pouvoir-faire : l'action est

de deux artefacts porteurs de valeur, les artefacts évaluatifs étant constitués par l'appréciation après coup de la transaction marchande comme dans les sites de vente en ligne.

14 H.A. Simon (1973) a introduit les termes de rationalité substantive et procédurale dans une acception différente mais pas totalement indépendante de celle que nous donnons à ces termes : «Le comportement est substantivement rationnel quand il est en mesure d'atteindre les buts donnés à l'intérieur des limites imposées par les conditions et les contraintes données (...) Une fois ces buts fixés, le comportement rationnel est entièrement déterminé par les caractéristiques de l'environnement dans lequel il a lieu.». 
justifiée en tenant compte de la manière dont les conditions de réalisation (connaissances, situation, contexte...) contribuent à la performance, celle-ci étant même en partie définie par la qualité du processus de réalisation.

- Rationalité agentive ${ }^{15}$ et orientation de l'activité selon l'axe du devoir : l'action est justifiée en tenant compte de la manière dont l'artefact porteur de valeur sert principalement à construire les relations entre destinateur (mandant) et destinataire, sujet réalisateur et sujet bénéficiaire, la performance étant également définie par la qualité des liens créés par le processus de réalisation.

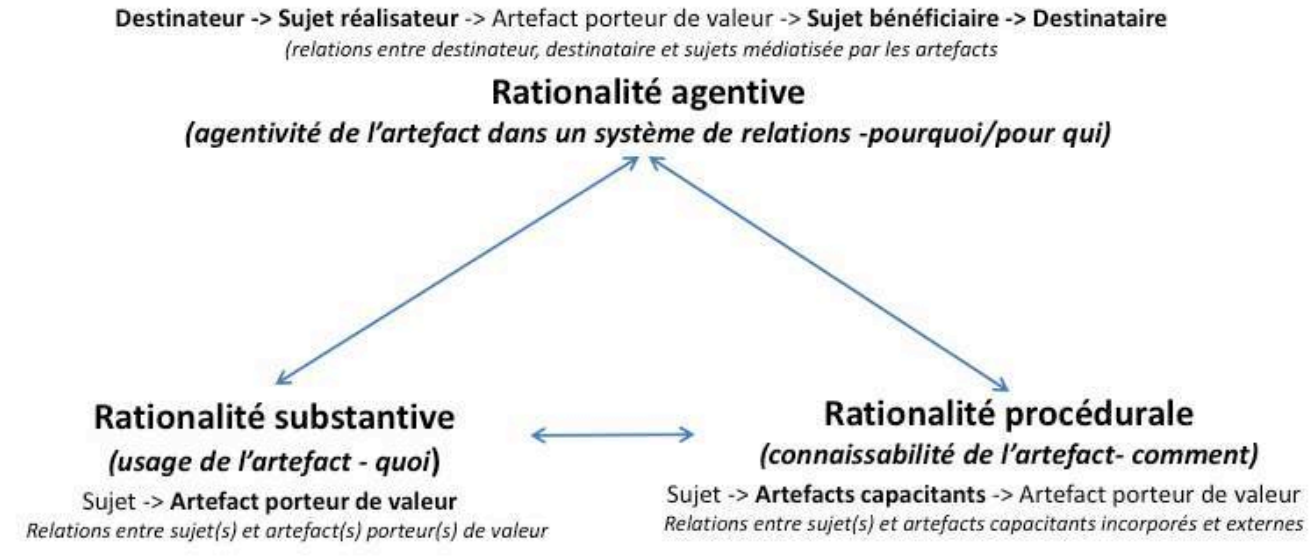

Figure 5 : Trois modalités de structuration des relations actancielles et de rationalisation

Figure 5: Three modalities of structuring actantial relations and rationalization

La référence à la logique des relations de Pierce (cf. Steiner, 2013) permet de mettre en relief l'association entre les catégories de Pierce et les actants de Greimas: l'identification de la valeur de l'objet relève d'une logique monadique, les savoir-faire permettant de construire ou d'utiliser l'artefact, d'une logique binaire et la définition de la manière dont l'artefact médiatise les relations entre les participants d'une logique ternaire (Tableau 6).

Ces différents registres de structuration des relations actantielles et de problématisation de la situation d'enquête peuvent également se comprendre comme des formes de lectures des situations renvoyant à différentes rationalités. On pourra penser aux distinctions devenues classiques proposées par Max Weber entre (1) actions affectives, guidées par l'émotion et les sentiments, (2) traditionnelles, guidées par l'habitude, (3) rationnelles en valeur, que cette valeur soit éthique, esthétique ou religieuse et enfin, (4) rationnelles instrumentalement, l'environnement et les tiers étant appréhendés comme des moyens intervenant dans l'atteinte des buts (Weber, 1978, pp. 24-25). On pourra également évoquer les approches de T. Parsons et son collègue le psychologue social R. F. Bales (Parsons, Bales, \& Shils, 1953) qui définissent quatre problèmes fonctionnels que doivent gérer les groupes et les individus synthétisés dans le modèle AGIL : adaptation opérationnelle (économie), atteinte des buts (système politique), intégration (média, religion), fonction latente de maintien de la structure (éducation). Une des sources de ce modèle réside dans les observations empiriques de l'activité de groupes restreints dans lesquelles ces différentes fonctions sont prises en charge par des interventions principalement verbales de différents intervenants au fur et à mesure du déroulement de l'activité.

15 Sur la notion d'agentivité voir, par exemple, Robichaud (2006). 


\begin{tabular}{|l|l|l|}
\hline $\begin{array}{l}\text { Priméité, quoi, logique } \\
\text { monadique, rationalité } \\
\text { substantive (vouloir) }\end{array}$ & Valeur_d_usage(AM_porteur de_valeur) & $\begin{array}{l}\text { Est_désirable (repas) } \\
\text { Est_savoureux (repas) }\end{array}$ \\
\hline $\begin{array}{l}\text { Secondéité, comment, } \\
\text { logique binaire, } \\
\text { rationalité procédurale } \\
\text { (pouvoir) }\end{array}$ & $\begin{array}{l}\text { Valeur_travail (artefact_capacitant, } \\
\text { AM_porteur_de_valeur) }\end{array}$ & $\begin{array}{l}\text { Composé_de (protéines, repas) } \\
\text { Permet_cuisson (four, repas,) } \\
\text { Donne_gout (faire_sauce, } \\
\text { repas) }\end{array}$ \\
\hline $\begin{array}{l}\text { Tiercéité, pour } \\
\text { qui/pourquoi, logique } \\
\text { ternaire, rationalité } \\
\text { agentive (devoir) }\end{array}$ & $\begin{array}{l}\text { Valeur_d'échange/ conventionnelle } \\
\text { (bénéficiaire/destinataire, realisateur/ } \\
\text { destinateur, AM_porteur_de_valeur) }\end{array}$ & $\begin{array}{l}\text { Demande_à (mère,grand_père, } \\
\text { repas) } \\
\text { Produit_au_bénéfice_de_par } \\
\text { (repas, enfant, grand_père) }\end{array}$ \\
\hline
\end{tabular}

Tableau 6 : Modalités de rationalisation de l'activité selon la logique des relations de Pierce

Table 6: Modalities for the rationalization of activity according to Peirce's logic of relations

Plus près de nous, notre conception est proche de celle que nous défendions dans la théorie des transactions intellectuelles (Zacklad, 2000) en nous appuyant sur le modèle des apprentissages croisés d'A. Hatchuel (1996). Selon cette théorie "L'articulation entre la nature des relations et la nature des savoirs est une tension fondamentale de l'action collective. » (Hatchuel 1996, p. 105). Tout développement de savoir-faire retentit sur les relations entre les actants humains et réciproquement toute transformation du système de relations affecte les connaissances mobilisables.

En adoptant la perspective que nous défendons ici, il faut rajouter la définition des caractéristiques de l'artefact porteur de valeur : le «but» à atteindre ou «l'objet » à concevoir définit, du point de vue du désir, des propriétés correspondant à des fonctions d'usage, dont toute évolution impacte simultanément les moyens et les relations de manière symétrique. Toute évolution du contour fonctionnel de l'artefact porteur de valeur impacte les connaissances et les technologies mobilisables et symétriquement, toute évolution des ressources cognitives et technologiques disponibles impacte ce contour fonctionnel (dimension décrite dans la théorie CK, Hatchuel \& Weil, 2002). Du point de vue des relations, une évolution des attentes et des besoins des destinataires et des bénéficiaires transforme le périmètre de la valeur d'usage et symétriquement une évolution de ce périmètre impacte les modalités d'engagement et les relations entre les acteurs impliqués dans la co-production et/ou la consommation de l'artefact porteur de valeur.

\section{3.- Activités de contrôle de la cohérence de l'expérience}

Le troisième axe d'analyse de l'activité, relève du contrôle de la cohérence de l'enquête et du programme transactionnel associé dont on a vu qu'elle pouvait être associée à la conduite d'un projet d'innovation, de conception, ou de changement organisationnel plus ou moins formel. Dans la philosophie pragmatiste, le contrôle de l'enquête est directement lié à des enjeux cohérence «logique» visant à proposer des résultats vérifiables, mais d'une logique instrumentale ancrée sur les activités de recherche pratiques des sujets et dont le modèle est davantage à chercher dans l'établissement d'une preuve par un tribunal que dans les raisonnements mathématiques purement formels (Dewey, 1938).

La logique de l'enquête relève pour l'essentiel d'une démarche expérimentale dans laquelle les expériences vécues sont confrontées à un corpus de connaissances établi (mais révisable) pour guider le déroulement de la transaction. Selon l'axe du contrôle, les activités d'enquêtes associées aux transactions inventives apparaissent pour une part comme relevant de démarches hypothético-déductives, mais au sens de l'épistémologie pragmatiste, pour laquelle la finalité de l'enquête est «la transformation contrôlée ou dirigée d'une situation indéterminée en une situation qui est si déterminée en ses distinctions et relations constitutives qu'elle convertit les 
éléments de la situation originelle en un tout unifié» (p. 169) et pas la production de connaissances générales pour «elles-mêmes ». Dans ce contexte, l'expérience ne vise pas comme chez Popper, prenant comme modèle exclusif la physique fondamentale, à déduire la généralité et la vérité d'un énoncé scientifique, mais «à en fonder le caractère opérationnel dans une situation particulière $\gg\left(\right.$ Gros, 2011) ${ }^{16}$.

Par ailleurs, les expériences auxquelles il est fait référence ne sont pas extérieures au sujet, mais l'impliquent directement. Il s'agit d'une vision «expérimentale de l'expérience », dans laquelle celle-ci «n'implique plus une posture passive, mais au contraire une mise à l'épreuve active et réflexive de la réalité et de nos connaissances (toujours provisoires) » (cf. introduction de G. Truc, Dewey 2008). Par ailleurs, «il ne suffit pas de faire une expérience : pour avoir de l'expérience, dirait-on trivialement, il faut avoir vécu, c'est-à-dire qu'il faut aussi avoir souffert, avoir enduré les conséquences de ce qu'on a fait » (ibid.). Cette vision de l'expérience la rapproche de la problématique de l'engagement développée par la sociologie pragmatiste même si celle-ci ne se réclame pas toujours de cette épistémologie. L. Thévenot (2006) dans sa description d'une pluralité de « régimes d'action » décrit ainsi différents régimes d'engagement, en familiarité, en plan et en justification. Cette dimension de l'engagement dans les situations peut donc être rapprochée de la manière dont la transformation contrôlée des situations implique des expériences actives de la part des sujets pour garantir la vérifiabilité du résultat des enquêtes.

Alors que la rationalisation inscrit l'action dans différents registres de justification, non pas antagonistes, mais complémentaires et symétriques (substantive, procédurale et agentive), le contrôle vise à garantir la cohérence et la vérifiabilité des démarches tout au long du programme transactionnel et de ses épisodes : virtualisation, acquisition de compétence, coréalisation de la performance, évaluation. En écho lointain avec les régimes d'engagement de la sociologie pragmatique nous analysons les activités de contrôle en référence à trois niveaux de description et d'expérience des situations, deux niveaux de description existentiels, en singularité et en généricité et le troisième en universalité. Ces différents niveaux de description et d'engagement dans les programmes transactionnels sont complémentaires et nécessaires pour raisonner de manière vérifiable et légitime, c'est-à-dire pour réaliser "l'ensemble des opérations qui consistent à faire l'examen des hypothèses suggérées et de leurs implications possibles » (Gros, ibid.). Mais selon les collectifs, les organisations, les styles personnels, on pourra noter une préférence pour certains niveaux de contrôle.

La référence à ces trois niveaux de description des propositions et de l'expérience s'appuie sur la logique de l'enquête de Dewey dont nous avons systématisé certains éléments lors d'une précédente recherche dans le domaine de l'ingénierie des connaissances (Zacklad, 1993). À chaque niveau, il est possible de décrire les connaissances mobilisées sous forme de termes reliés par des propositions pouvant correspondre à des règles conditionnelles. Ces propositions peuvent relier des termes de même niveau de description ou permettre le passage d'un niveau de description à l'autre au fur et à mesure du raisonnement (Zacklad, 1993, p. 69) pour rendre compte de la manière dont s'organisent les raisonnements en situation, la montée en généralité ou, au contraire, l'application de connaissances générales à des cas particuliers.

Il est possible d'assimiler l'ensemble de ces propositions à des règles quel que soit le niveau de description choisi (singulier, générique, universel) en rejoignant ainsi la terminologie de la sociologie du travail (par ex. Reynaud 1989) ou de la théorie de la structuration (Gidden,s 1984) comme il est possible de considérer que les règles sont un niveau d'expression particulièrement adapté à la description de connaissances génériques comme nous l'avons fait dans d'autres textes (cf. Zacklad, 2007b) en parlant de régulation basée sur les règles pour décrire le contrôle en généricité. Cette utilisation du terme de règle pour décrire un niveau de contrôle spécifique est aussi celle de J. Rasmussen (1983), auteur célèbre en psychologie ergonomique, qui

16 Voir aussi G. Truc (Dewey, 2008) son introduction de l'article de Dewey, « La réalité comme expérience ». 
distingue trois niveaux de régulation de l'activité, basés sur les habiletés, les règles et les connaissances générales. Ici, nous prendrons l'option terminologique inverse, en assimilant les règles à des formes de description de l'expérience quel que soit son niveau d'expression en singularité, en généricité ou universalisant (Figure 6).

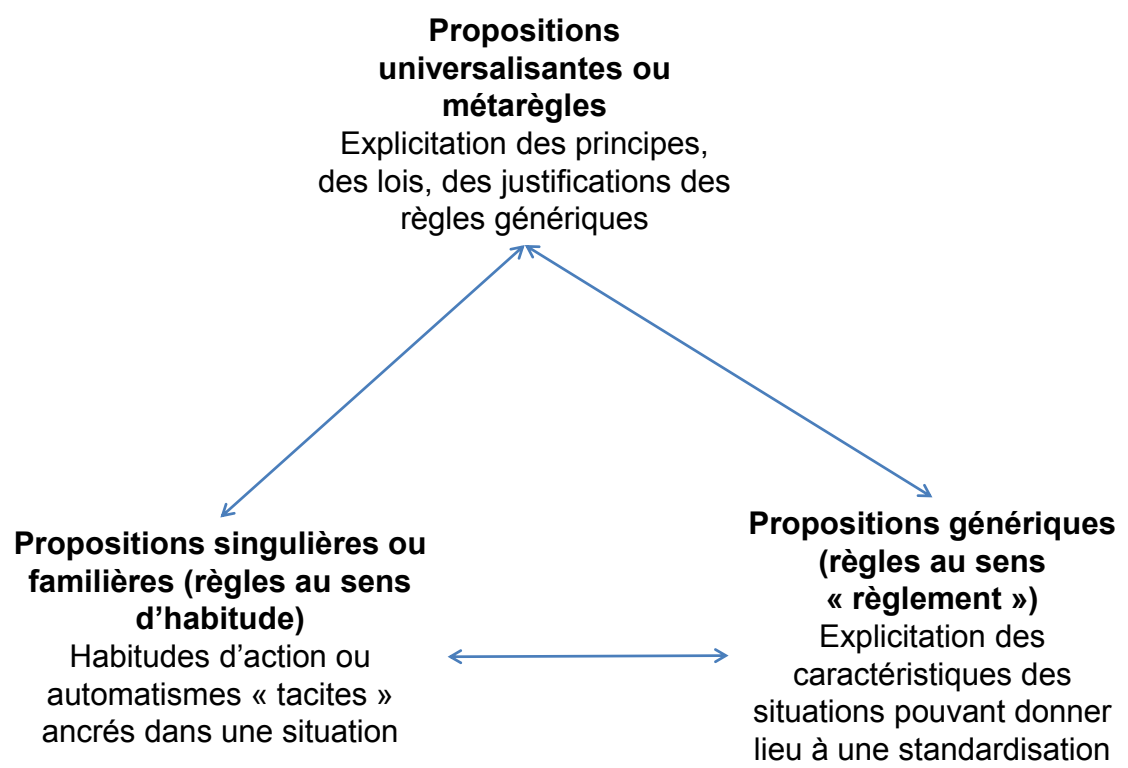

Figure 6 : Modalités de contrôle de la cohérence des enquêtes

Figure 6: Modalities for controlling inquiry consistency

La différence entre les activités de contrôle et d'expérimentation existentielles et universelles est que les deux premières cherchent à maintenir un lien permanent avec les caractéristiques des situations de référence tandis que la troisième, universelle, s'appuie sur des objets théoriques ou fictionnels pour énoncer des principes qu'il faut impérativement particulariser pour être en mesure de les opérationnaliser. Ces entités générales peuvent être des lois de nature scientifique ou relever de la référence à des biens communs dans un registre plus politique. Une expérience dans le registre de l'universel est d'abord une expérience de pensée et d'imagination qui s'éprouve à l'aide d'artefact sémiotique dans le cadre d'échanges discursifs plus ou moins interactifs avant de guider un processus de transposition dans la sphère existentielle.

Dans le domaine existentiel, le contrôle en singularité est directement ancré dans les propriétés singulières des situations. À ce titre, il s'appuie de manière préférentielle sur des informations sensori-motrices, issues de la manipulation comme de la perception d'artefacts tangibles. Les règles relevant du contrôle en singularité sont ancrées dans l'habitude et les routines, leur formulation linguistique s'appuyant largement sur des expressions indexicales. Plus que tout autre, le contrôle en singularité implique que les sujets fassent l'expérience directe des situations de référence. Cette notion de proximité se retrouve dans le régime de l'engagement en familiarité ou engagement «en proche » de L. Thévenot qui insiste pour sa part sur l'aise que l'on ressent dans les environnements connus «où l'on s'y retrouve les yeux fermés, par une sorte de pleine disponibilité et de pleine convergence entre le corps, les êtres et les objets » (Genard, 2011).

Le contrôle en généricité résulte d'un effort de généralisation qui vise à systématiser la description et la formulation des règles en s'appuyant sur les caractéristiques communes des situations et de l'expérience. Cet effort de systématisation et de généralisation commence par la définition d'une terminologie stabilisée comprenant des noms communs et des noms propres. De ce fait, les règles peuvent être comprises par des collectifs plus vastes que ceux qui se constituent dans des pratiques routinières communes dans un environnement spatiotemporel 
directement partagé. En contrepartie, les règles génériques requièrent des activités interprétatives pour être mises en œuvre en situation. C'est la formulation générique des connaissances qui permet une standardisation du contrôle et de l'expérience en explicitant les caractéristiques des artefacts et des personnes qu'il faut prendre en compte. Les règles génériques peuvent être partie intégrante des plans et des contrats ce qui suggère des parallèles avec le régime des engagements en plan de L. Thévenot.

Mais la référence aux situations et à leurs propriétés, même exprimée de manière plus générale, implique toujours une forme de stabilité du contexte pour être mise en œuvre. Or, dans toute une série de cas, cette stabilité fait défaut du fait de l'inventivité des acteurs, de la diversité des environnements, ou de la transformation du contexte de l'action sous l'effet de facteurs extérieurs. Dans ce cas, les activités de contrôle recourent à une autre forme de montée en généralité dite en universalité. Les propositions universelles qui font appel à des entités abstraites décrivent des modes d'opération possibles et sont intrinsèquement hypothétiques. Dans le domaine juridique et social, elles correspondraient à des lois ou au recours à des principes supérieurs communs au sens de Boltanski et Thévenot (1991) et elles renvoient au régime d'engagement en justification de ce dernier auteur. Mais elles peuvent aussi s'exprimer de manière narrative comme dans les paraboles. Elles sont associées à des pratiques d'argumentation et de narration plutôt qu'à la confrontation à des situations pratiques engageant la manipulation d'artefacts situés.

Chacun de ces régimes de contrôle est nécessaire pour garantir une transformation cohérente des situations durant l'enquête. Le contrôle en singularité garantit l'opérationnalisation effective de la performance dans des situations concrètes par la prise en compte des corps et des dimensions tangibles de l'environnement. Le contrôle en généricité permet de déployer l'activité dans des territoires et des collectifs plus large (Zacklad, 2007b) en étant également susceptible d'apporter plaisir et confort. En effet, la généricité et la standardisation qu'elle rend possible peuvent permettre de découvrir de nouveaux environnements et de nouveaux collectifs analogues en fournissant de l'assurance face à la nouveauté. Enfin, le contrôle universalisant qui vient suppléer aux défaillances du contrôle en généricité dont la cohérence peut être mise à mal (Zacklad, 2007b) est l'occasion pour les acteurs de déployer de nouvelles formes de créativité correspondant à la définition de «monde nouveau » porteur de représentations et d'émotions inédites.

Cadrage, recadrage, conflit de cadrage : management et travail de contrôle

Nous sommes maintenant en mesure de revenir sur la problématique des activités de cadrage du contexte et de les mettre en relation avec le travail de management. Le travail de management tel que nous l'envisageons ici est un travail qui concerne potentiellement tous les opérateurs et qui correspond en partie à ce que certains sociologues nomment le «travail d'organisation » (cf. supra), un terme qui a pour nous l'inconvénient de renvoyer essentiellement à la gestion des relations entre les personnes au détriment des dimensions plus techniques de l'activité, un défaut que possède également l'expression de «management» si on l'envisage dans un sens restreint. Le management peut concerner sa propre activité auquel cas ces activités relèvent de ce qu'en ergonomie, P. Falzon nomme des activités méta-fonctionnelles :

"Il s'agit d'activités non directement orientées vers la production immédiate, activités de construction de connaissances ou d'outils (outils matériels ou outils cognitifs), destinée à une utilisation ultérieure éventuelle, et visant à faciliter l'exécution de la tâche ou à améliorer la performance.» (Falzon, 1994).

Mais selon nous, les activités de management, pilotage et cadrage, peuvent aussi être envisagées comme des activités faisant l'objet d'une performance spécifique distincte de la performance de la transaction qu'elles visent à piloter ou à cadrer. Elles relèveraient à ce titre d'un travail inscrit dans une transaction secondaire dont l'artefact porteur de valeur est la performance d'une transaction primaire. Dans le domaine du CSCW, en particulier chez Schmidt et Simone (1996), on trouve la notion de «travail d'articulation» (articulation work) qui est évoquée strictement 
dans le contexte de la coordination des tâches dans un travail coopératif relevant d'une coproduction matérielle pouvant exclure toute intentionnalité. Ce travail d'articulation, qui peut lui-même être réalisé en coopération, est un exemple de transaction secondaire dont les bénéficiaires sont les opérateurs engagés dans la transaction primaire productive (dans un sens matériel). La sémiotique des transactions coopératives est, quant à elle, plus ouverte aux situations de travail immatérielles et relationnelles dans lesquelles le travail correspond à une communication transformative (Zacklad, 2012b).

Le travail de management correspond aux activités de contrôle de la cohérence de l'expérience que nous avons présentées dans la partie précédente quand celles-ci ont un caractère systématique. Ces activités mobilisent souvent des analyses réflexives. Les activités de contrôle ne relèvent à proprement parler du management que quand elles sont réalisées de manière consciente et délibérée dans le cadre de transactions finalisées orientées par une performance. Nous établirons ici une distinction entre deux types d'activité de management, les activités de pilotage et celles de cadrage (cf. Tableau 7).

\begin{tabular}{|l|l|l|}
\hline & $\begin{array}{l}\text { Application des règles dans une } \\
\text { situation routinière (= pilotage de la } \\
\text { situation) }\end{array}$ & $\begin{array}{l}\text { Création de nouvelles règles dans une } \\
\text { situation d'enquête (= cadrage du } \\
\text { contexte) }\end{array}$ \\
\hline Pour les autres & Pilotage managérial & Cadrage managérial \\
\hline Pour soi & Pilotage de sa propre activité & $\begin{array}{l}\text { Cadrage de sa propre activité (activité } \\
\text { méta-fonctionnelle) }\end{array}$ \\
\hline
\end{tabular}

Tableau 7 : Pilotage et cadrage managérial

\section{Table 7: Managerial monitoring and framing}

Le pilotage intervient dans des situations routinières qui mobilisent un système de règles existant qu'il faut adapter à la situation transactionnelle courante. Par contraste, le cadrage intervient dans les situations d'enquête qui sont beaucoup plus incertaines. Il implique la mobilisation de règles originales en situation de résolution de problème, que ces règles soient inspirées de situations préexistantes dans le cas du recadrage, ou qu'elles soient authentiquement originales dans le cadrage inventif (cf. infra). À ce titre, le cadrage est susceptible d'impacter le contexte de la transaction, c'est-à-dire de transformer de manière relativement durable les milieux humains et techniques dans lesquels celle-ci se déroule et se prolongera le cas échéant.

Le cadrage peut concerner la gestion temporelle et l'entretien de la motivation (virtualisation, compétence, performance, évaluation) ou les différents registres de la rationalisation (substantive, procédurale, agentive). Par ailleurs il peut être appréhendé en physicalité comme en intériorité. Quand il est appréhendé en physicalité et du point de vue des artefacts, il se traduit par la mise en œuvre d'artefacts tangibles qui vont contraindre matériellement l'action : contrôle de la perception et des déplacements ou contrôle des activités d'écriture dans les supports numériques (cf. la délégation du contrôle aux artefacts dans l'analyse des «gendarmes couchés » ou ralentisseurs par Latour, 1996). Quand il est appréhendé en intériorité, il correspond le plus souvent à l'usage d'artefacts à dominante sémiologiques intégrés dans des formations discursives.

Le cadrage contextuel et le travail de management peuvent être réalisés par les participants à la transaction ou par des prescripteurs, et ce de manière délibérée ou de manière tacite. Dans les organisations productives contemporaines marquées par la bureaucratisation et le taylorisme, une part importante du cadrage contextuel de l'action collective est issue des productions délibérées de managers professionnels plus ou moins impliqués dans la vie des services: qualité, contrôle de gestion, système d'information, etc. qui visent à spécifier les artefacts médiateurs, les technologies et les rôles des actants humains. D'autres éléments de cadrage sont 
issus de la formation professionnelle des sujets et sont régulièrement actualisés par la participation à des séminaires et à des rencontres d'échanges de pratiques dans le cadre de l'entretien de la professionnalité. Mais les sujets eux-mêmes cadrent leur pratique, soit de manière tacite, soit en produisant de manière délibérée des règles de contrôle leur permettant de gérer le déroulement temporel de l'activité et de la justifier (rationalisation).

\section{4.- Cadrage et conflits de cadrage du contexte transactionnel dans la conduite du changement}

Dans cette dernière partie, nous illustrerons certaines dimensions du travail de management en présentant un processus de cadrage et de recadrage du contexte transactionnel au sein d'une organisation. Celui-ci s'inscrit dans ce qu'il est convenu d'appeler un projet de conduite du changement porté par le management qui a donné lieu, comme c'est souvent le cas, à des phénomènes de résistance à ce changement qui ont porté à la fois sur le cadrage des étapes du programme transactionnel, orientation motivationnelle (vision stratégique, compétences clefs, définition de la performance, modalités d'évaluation) et sur les modalités de rationalisation (substantive, procédurale et agentive).

Ce cas s'inscrit dans le mouvement de réforme des universités françaises, la LRU, qui en échange d'une autonomie accrue, doivent se plier à nouvelles règles budgétaires liées à la mise en place d'une réforme de la comptabilité publique (la LOLF), à une évolution du statut des personnels et à une nouvelle gouvernance. L'arrivée dans l'établissement étudié d'un nouveau directeur ${ }^{17}$ possédant une expérience importante de l'entreprenariat dans le secteur privé a donné lieu à un projet majeur de changement organisationnel visant à adapter l'organisation à ces nouvelles règles (Tableau 8 ).

\begin{tabular}{|l|l|}
\hline Actants génériques & Actants dans le cas de l'université \\
\hline Artefact porteur de valeur & $\begin{array}{l}\text { Fonctionnement de l'université selon un modèle matriciel } \\
\text { inspiré du privé }\end{array}$ \\
\hline Artefacts capacitants qualifiant & $\begin{array}{l}\text { Connaissances en comptabilité, en gestion de projet, en } \\
\text { management des hommes, documentation et logiciels associés }\end{array}$ \\
\hline Sujet coréalisateurs et bénéficiaires & $\begin{array}{l}\text { Les personnels, enseignants-chercheurs, administratifs, } \\
\text { techniciens }\end{array}$ \\
\hline $\begin{array}{l}\text { Destinateur (personne motivant et } \\
\text { initiant le programme transactionnel) }\end{array}$ & Le directeur de l'université \\
\hline Destinataires & $\begin{array}{l}\text { Le ministère de tutelle, les étudiants, les personnels adhérents } \\
\text { au projet de réforme }\end{array}$ \\
\hline
\end{tabular}

Tableau 8 : Quelques actants clefs dans le projet d'autonomie de l'université

Table 8: Some key actants in the university's autonomy project

\section{1.- Cadrage stratégique (virtualisation) et rationalisation substantive}

Le recadrage stratégique s'appuie souvent sur une rationalisation substantive dans laquelle on considère que l'activité est essentiellement pilotée en orchestrant l'adhésion à des buts indépendamment de la manière dont les processus qui permettront de les atteindre conditionnent leur définition. La rationalisation substantive est essentiellement organisée autour de la définition de «visions » et de critères de valeur qui permettront d'établir une différenciation claire entre fins et moyens, les seconds étant entièrement subordonnés aux premiers. Il est donc assez classique que dans l'étape de virtualisation stratégique, ce type de rationalisation soit privilégiée, même si la rationalisation agentive, qui explicite la relation aux commanditaires et aux bénéficiaires peut également être de mise.

17 Dans cette université possédant un statut dérogatoire, le directeur est nommé par le CA. 
C'est d'abord au sein du comité de pilotage composé de membres influents de l'organisation que s'est élaborée la nouvelle vision de la mission de l'université et de son environnement justifiée par la doctrine gouvernementale de l'autonomie des universités. La mise en place d'un discours managérial en partie exogène à l'institution considérée a été au cœur de l'introduction du changement. Les objectifs annoncés visaient une meilleure transversalité, une meilleure réactivité, un suivi budgétaire performant, une mobilité des personnels accrue, etc. Une caractéristique corollaire de ce projet et de l'enquête, au sens pragmatiste, auquel il a donné lieu, est de ne pas être parti de dysfonctionnements constatés de manière empirique sur le terrain, mais plutôt de principes généraux quasiment théoriques, s'appuyant à la fois sur les prescriptions gouvernementales et leur inspiration proche des principes du New Public Management et sur des représentations managériales issues de l'entreprise privée. Dans nos termes, il s'agissait d'une modalité de contrôle de l'action de type universalisant, l'enquête visant principalement, pour le management, à expérimenter l'applicabilité de ces principes au contexte de l'université pour, dans un second temps, en évaluer les bénéfices internes et externes.

Si ce projet de changement a donc été initié par des formations discursives universalisantes, la « résistance au changement» s'est également exercée sur ce plan. En l'occurrence, selon les mêmes modalités, des arguments contradictoires également universalisants et relevant d'une rationalité substantive et agentive, ont fait appel au registre civique dans l'opposition classique, mais souvent stérile, entre missions de service public et critères de rentabilité relevant du registre marchand. Les autres formes de résistance, plus subtiles, se sont appuyées sur des formes de rationalisation procédurales, invoquant les caractéristiques spécifiques des professions exercées par les enseignants-chercheurs, leurs modes de recrutement, de promotion, de reconnaissance, peu susceptibles de se couler dans le moule classique du management et de l'évaluation de la performance du secteur industriel ou des services soumis à des normes de gestion dominées par des critères de performance substantifs d'ordre financier.

\section{2.- Cadrage de la compétence en rationalité procédurale et agentive}

Dans les organisations professionnelles, le cadrage de la compétence passe souvent assez logiquement par les plans de formation. Dans notre cas, les plans de formation ont occupé une place importante dans le projet de réforme, avec l'objectif assumé de produire une transformation des représentations et en choisissant des modules de formation se revendiquant parfois eux-mêmes d'un projet de «modernisation». Selon une autre perspective, la formation part de la pratique et les formateurs sont eux-mêmes amenés à concevoir des programmes ad hoc directement inspirés des problématiques de l'entreprise.

C'est surtout la hiérarchie intermédiaire qui a été ciblée, et ce dans deux principales directions : les connaissances instrumentales dans le domaine de la comptabilité et de la gestion, profondément impacté par la réforme (LOLF) et la formation au «management», au sens restreint de ce terme désignant l'animation et la motivation des équipes. En ce qui concerne les connaissances en gestion, l'ensemble des personnels du service financier qui a dû revoir ses méthodes de travail a suivi les formations dispensées, comme, il est vrai, une grande partie des cadres en comptabilité et en finance de l'administration française. Mais d'une certaine manière, les résultats n'ont pas été à la hauteur des attentes de la direction qui a mis en place un service ad hoc dédié au contrôle de gestion et composé pour l'essentiel d'agents contractuels venus du secteur privé.

Les formations au management visaient principalement les enseignants-chercheurs qui en faisaient la demande parmi ceux nommés aux postes de responsables de programmes de recherche et d'enseignement et aux postes de responsables de pôles de compétence. Elles s'adressaient également à certains personnels administratifs responsables d'équipe ou de service. Dans ce contexte, la résistance au cadrage s'est manifestée à travers un refus de s'inscrire aux formations, où à l'intérieur des formations elles-mêmes, sous la forme d'une 
contestation des contenus, notamment dans les formations dites managériales. Alors que les formations en gestion relevaient d'une rationalisation principalement substantive et procédurale, les formations dites «managériales» (au sens restreint) relevaient essentiellement d'une rationalisation agentive visant à cadrer la nature des relations à l'intérieur des services et entre les services en termes de contractualisation et de style de communication, de manière à développer les capacités à rendre compte et à entretenir les relations et la motivation.

\section{3.- Cadrage de la performance organisationnelle et rationalisation agentive}

La principale transformation a concerné le recadrage de la performance qui a fait l'objet d'un contrôle de cohérence actif à tous les niveaux. La performance dont il est question ici est liée à la co-production de la valeur à l'intérieur de l'organisation, dans la coopération entre les membres de l'organisation plutôt que vis-à-vis des bénéficiaires externes : étudiants, partenaires de recherche, entreprises recrutant les stagiaires, etc. Bien sûr, la performance organisationnelle doit permettre d'atteindre une performance externe vis-à-vis de ces bénéficiaires. Mais dans un premier temps, l'accent a été mis sur la performance interne dans le cadre des hypothèses universalisantes issues du projet de réforme étatique déjà évoqué, la direction considérant a priori que la mise en place d'un fonctionnement organisationnel différent conduirait à une amélioration de la performance externe.

La description dans la perspective de la performance met l'accent sur la manière dont l'artefact porteur de valeur est le produit et l'objet des relations entre réalisateurs et bénéficiaires : production, co-production, transfert, réception, consommation, etc. Une vision substantive de la performance privilégie la définition des caractéristiques de l'artefact porteur de valeur ou de ses avantages pour le bénéficiaire. Une vision procédurale privilégie les connaissances, savoir-faire, savoir-être, procédures, méthodes associées à la production de l'artefact porteur de valeur ou, le cas échéant, à sa réception. Enfin, la perspective de la rationalité agentive met l'accent sur la manière dont la médiation de l'artefact porteur de valeur cadre les rôles et les attentes des acteurs qui sont impliqués dans l'action. Cette définition des rôles, des relations et des prérogatives associées se retrouve notamment dans l'organigramme, que celui-ci mette l'accent sur les relations hiérarchiques ou sur les modalités prescrites de circulation des informations entre les acteurs.

$\mathrm{Au}$ lieu d'une organisation classique par départements d'enseignement et laboratoires, la réforme visait à introduire une organisation matricielle croisant des responsables de "pôles de compétence » et des responsables de programmes de recherche et d'enseignement. Cette réforme a été d'abord largement justifiée de manière universalisante sur la base des principes de transparence et d'efficience déjà évoqués et promus par le comité de pilotage. Elle a fait ensuite l'objet d'un effort déploiement intense en généricité qui a impliqué de nombreuses réunions consacrées à la formalisation des nouvelles fiches de postes et des procédures administratives. Les postes d'assistantes et de secrétaires ont été particulièrement concernés.

Le contrôle s'est exercé en intériorité par la promotion de règles de fonctionnement et en physicalité, par la mise en place d'un vaste plan de déménagement visant à mettre en cohérence les relations décrites dans l'organigramme et la proximité physique des personnes dans les bâtiments. Dans ce plan de déménagement, la primauté a souvent été donnée à la structure «pôle » par rapport aux « anciens » laboratoires et départements. Le contrôle en singularité a également fait l'objet d'une attention particulière. Chaque service a été mis en conformité avec les règles génériques en tenant compte autant que possible de sa spécificité, mais en cherchant également à rompre délibérément avec des habitudes de travail jugées non conformes aux règles (généricité) ou à l'esprit de la réforme (universalité).

La résistance à cette nouvelle forme de cadrage s'est traduite par un refus de la part de certaines personnes d'accepter les nouveaux postes pourtant considérés comme prestigieux dans la nouvelle structure, ou par une acceptation suivie d'une forme d'exercice des responsabilités seulement partiellement conforme à leur définition initiale. Dans le registre du cadrage en 
intériorité, la résistance s'exerce par la mise en place d'un double langage, les enseignantschercheurs, voire leurs responsables directs, continuant de se référer au quotidien aux anciennes formes d'organisation en refusant d'utiliser les nouvelles dénominations proposées, attachement à l'appellation en termes de «laboratoire », par exemple. Enfin, en physicalité, certains documents numériques n'ont pas été remplis selon les normes attendues ce qui a impliqué parfois une adaptation des formulaires pour les adapter aux détournements d'usage quand ceuxci étaient prévalents.

\section{4.- Cadrage évaluatif à partir de rationalisations procédurales et substantives}

Le cadrage évaluatif a été promu dans le registre procédural essentiellement en généricité. Quand l'évaluation se déroule selon une rationalité procédurale, l'accent est mis sur les manières de faire, savoir, savoir-faire, savoir-être mobilisés, techniques employées, étapes suivies. Les nouveaux indicateurs de suivi de l'activité mis en place dans le domaine de l'enseignement (p.e. durée des thèses) ou de la recherche (volume de contrats) nous semblent ainsi relever d'une rationalité procédurale. D'autres formes d'évaluation relevant d'une rationalisation substantive ont également été mises en place, comme les entretiens annuels d'évaluation dans lesquels on cherche à estimer l'atteinte des objectifs, mais également à discuter les projets d'évolution ou de mobilité du personnel.

Un grand nombre d'indicateurs ont été par ailleurs créés pour répondre à des besoins d'évaluation correspondant aux attentes de l'autre destinateur principal de la réforme, le ministère de tutelle, dans le cadre du projet d'autonomie de l'établissement plutôt inscrit dans une perspective substantive. En physicalité, la plupart de ces indicateurs ont donné lieu à un projet de recueil informatisé, souvent par le biais de fichiers de tableur impliquant la saisie de données le plus souvent quantitatives.

La résistance à ce cadrage évaluatif s'est effectuée par un refus de se conformer à certaines prescriptions ou à une forme d'exercice simplifiée à l'extrême. À ce propos, on remarquera que dans la plupart des cas, l'évaluation s'exerce en généricité en recourant à des formulaires déclaratifs et ne tient que peu compte de la diversité des situations (singularité) qu'il faudrait être en mesure d'observer de manière spécifique alors que c'est uniquement à ce niveau que les hypothèses universalisantes peuvent être confirmées.

\section{5.- Discussion}

À l'issue de cette présentation nécessairement trop rapide, il est possible de se livrer à quelques remarques conclusives. On pourra remarquer d'abord un paradoxe fréquent dans ce contexte de bureaucratie professionnelle. Alors que le cadrage de la performance est souvent fait dans les termes d'une rationalité agentive, par la définition de nouveaux postes et la redéfinition des fonctions et des relations, l'évaluation relève d'une rationalité substantive ou procédurale en généricité : la qualité des «résultats», pour autant que celle-ci puisse effectivement être estimée, ou les procédures suivies pour réaliser cette production. Ce n'est pas le «contenu » des relations et des expériences associées qui fait l'objet d'une évaluation, mais la manière dont les nouvelles transactions permettent d'aboutir à la production d'artefacts médiateurs généralement analysés en physicalité, en référence à des critères considérés comme mesurables et ce de manière assez standardisée, en généricité, comme peut l'être l'indicateur portant sur le volume annuel des contrats de recherche alors que le changement prescrit portait sur les formes de «management» des chercheurs induites par la nouvelle organisation. Si le passage d'une forme de rationalité à l'autre, de la prescription des modalités de réalisation de la performance à son évaluation, peut présenter un intérêt, le caractère purement externe des critères d'évaluation ne facilite pas forcément la compréhension des modalités d'obtention des résultats et donc l'apprentissage.

Cette dernière remarque n'est pas sans lien avec la seconde, relative à la difficulté rencontrée 
par ces organisations à cadrer le travail en intériorité et en singularité, c'est-à-dire à parler du contenu du travail sur la base de cas particuliers qu'il s'agisse, dans notre cas, du travail d'enseignement ou du travail de recherche. Cette problématique est récurrente dans le domaine du travail dit «immatériel et relationnel»(Du Tertre, 2005). Les évaluations se déroulent souvent en physicalité, à travers la mise en place de logiciels contraignant les activités informationnelles et communicationnelles, telles que les progiciels de gestion intégrés qui visent à recueillir des données objectives, dont le fonctionnement est stéréotypé, peu adaptable aux situations singulières et dont le paramétrage est inaccessible à ceux, opérateurs ou managers spécialisés, qui sont réellement en responsabilité de l'activité. Cette situation est d'autant plus paradoxale que ces organisations sont généralement prolixes en termes de production de discours universalisants invoquant des principes généraux, que ceux-ci émanent des prescripteurs ou des membres engagés dans une démarche de contestation.

\section{5.- Conclusion : un management pragmatiste ?}

À travers ce cas, nous espérons avoir contribué à éclairer certaines caractéristiques centrales du travail de management ou activité d'encadrement, qui consiste précisément à cadrer le contexte transactionnel de manière délibérée. Cette activité peut être mise en œuvre par du personnel d'encadrement au sens statutaire, par des leaders émergents dans des groupes restreints, des communautés de pratique ou d'action, ou par le collectif de manière collégiale. Elle décrit également l'activité individuelle dans des transactions où destinateur/réalisateur et bénéficiaire/destinataire peuvent être la même personne. Inscrire l'activité d'encadrement dans un continuum allant de la maîtrise de ses propres programmes transactionnels jusqu'à celle des collectifs dans lesquels l'individu est engagé, nous semble d'ailleurs également ouvrir des perspectives intéressantes en termes de formation et de gestion des ressources humaines.

L'approche transactionnelle du cadrage du contexte permet également de mettre l'accent sur les deux grandes dimensions du management correspondant, d'une part, à la planification dans le temps et à l'entretien de la motivation et d'autre part, à la rationalisation de l'action. La première dimension relève du cadrage du programme transactionnel. Dans la virtualisation, il y simultanément projection dans le futur, la futurité de Commons dont parle M. Renault (2013, dans ce numéro) et entretien de la motivation des sujets réalisateurs et/ou bénéficiaires pour les faire adhérer au programme transactionnel (vouloir). Mais la motivation doit être entretenue à toutes les étapes de la transaction en mettant l'accent sur le pouvoir conféré par l'acquisition des compétences, sur les bénéfices directs associés à la réalisation de la performance (acquisition et/ou production et/ou consommation) et enfin sur la rétribution et/ou reconnaissance faisant suite à l'évaluation de cette performance par le destinataire. La deuxième dimension relève de l'élaboration de rationalisations qui doivent soutenir le programme transactionnel tout au long de son déroulement. Le management doit être en mesure de fournir des justifications cohérentes et pertinentes au regard des actions effectives relevant de différents types de rationalités: substantive, la valeur des résultats, procédurale, la pertinence des méthodes, et agentive, les bénéfices internes et externes pour les destinataires directs et les parties prenantes.

Enfin, nous mettons l'accent sur les différents niveaux auxquels les activités de cadrage doivent s'exercer (1) au niveau du soutien au développement de routines et d'habitudes dont il n'est pas possible d'anticiper la forme définitive (2) au niveau de la formulation de règles facilitant le déroulement du programme transactionnel et les interprétations, correspondant à la mise en place de procédures (3) au niveau de l'élaboration de théorisations, notamment issues d'une analyse réflexive de l'activité, suivies d'un travail d'abstraction, qui permet de doter les acteurs d'une compréhension en profondeur des situations et d'accroître leur autonomie face à des évolutions importantes des conditions d'exercice de la pratique.

S'il y a bien également une vision pragmatiste du management, c'est celle qui consisterait à ne pas séparer le cadrage du contexte de la participation effective aux situations transactionnelles. En effet, quand il est délibéré, le cadrage du contexte part de l'expérience des situations pour y 
puiser les éléments susceptibles d'en faciliter la reproduction et l'amélioration. Dans les environnements de travail tertiaire inventifs qui sont notre cible, il est très difficile de procéder à une analyse objective externe de l'action de type taylorienne qui déboucherait sur un cadrage procédural exhaustif en généricité. Même quand celui-ci est possible, dans le travail dit d'exécution, la mise en œuvre exogène de règles génériques peu adaptables s'avère le plus souvent nuisible à la santé des opérateurs. Hors de ces cas limites, le management n'est légitime et pertinent que si les interventions de cadrage partent d'une connaissance personnelle des situations qu'il s'agit de cadrer issue d'une expérience directe ou de l'implication dans un travail réflexif associant les collaborateurs.

Pour utiliser la terminologie de la sociologie pragmatique de L. Thévenot (2006), il n'est possible d'être dans le registre du plan, de la mise en conformité, du cadrage délibéré du contexte transactionnel, qu'en s'appuyant sur des acquis dans le régime de l'action en familiarité. Quand cela n'est pas le cas, le contexte organisationnel apparait essentiellement comme limitant. Indépendamment de la difficulté à motiver l'action, les participants aux transactions ne parviennent pas non plus à lui donner du sens tant le décalage est grand entre les «theories in use », théories en usage issues d'un cadrage ascendant de la pratique et les « espoused theories », théories auxquels les acteurs adhèrent explicitement pour satisfaire à des injonctions exogènes, pour utiliser les termes d'Argyris et Schon (1996), des situations malheureusement encore fréquentes dans les organisations contemporaines.

\section{BIBLIOGRAPHIE}

Akrich, M., Callon, M., \& Latour, B. (Eds.) (2006). Sociologie de la traduction : textes fondateurs. Paris: Mines ParisTech, les Presses, « Sciences sociales »

Argyris, C., \& Schön, D.A. (1996). Apprentissage organisationnel. Théorie, méthode, pratique. Paris: DeBoeck Université.

Bidet, A., Boutet, M., \& Chave, F. (2013), Au-delà de l'intelligibilité mutuelle - Un apport de la tradition pragmatiste à l'étude des activités organisées. Activité, 10(1), dans ce numéro.

Boltanski L., \& Thévenot L. (1991). De la justification : les économies de la grandeur. Paris: Édition Gallimard.

Brassac, Ch. (2004). Action située et distribuée et analyse du discours : quelques interrogations, Cahiers de Linguistique Française, 26, 251-268.

Brassac, Ch. (2007). Qu'est-ce qu'un acte ? La réponse de George Herbert Mead. @ ctivités, 4(2), 165177.

Bruner, J. S. (1991). The narrative construction of reality. Critical Inquiry, 18, 1-21.

Clot, Y., \& Faïta, D. (2000). Genre et style en analyse du travail. Concepts et méthodes. Travailler, 4, 7 42.

Commons, J. (2001), Institutional Economics, paru dans The American Economic Review, 1931, no 4, traduit par L. Bazzoli et V. Dutraive, Cahiers d'économie politique, no 40-41.

Cooren, F. (1999). The organizing property of communication, Amsterdam/Philadelphia: John Benjamins Publishing Company.

Descola, P. (2002), Figures des relations entre humains et non-humains (suite), résumé des cours de l'année 2001-2002

http://www.college-de-france.fr/media/anthrop/UPL28452_UPL51939_DescolaR01_02.pdf (consulté le $15 / 08 / 2011)$

Descola, P. (2005), Par-delà nature et culture. Paris: Gallimard, Bibliothèque des sciences humaines.

Dewey J., (1938), Logic: The Theory of Enquiry, Henry Holt and Company, New York, traduction française (1993). Logique : La théorie de l'enquête, Paris: PUF.

Dewey J. (2008), La réalité comme expérience, Tracés. Revue de Sciences humaines [En ligne], 9 | 2005, mis en ligne le 11 février 2008, consulté le 21 août 2012. URL : http://traces.revues.org/204.

Dewey, J., \& Bentley, A. F. (1949). Knowing and the known. In J. A. Boydston (1989), John Dewey: The later works, 1925-1953 (Vol. 16, pp. 2-294). Carbondale: Southern Illinois University Press. 
Everaert-Desmedt, N. (1990), Le processus interprétatif. Introduction à la sémiotique de Ch.S. Peirce. Liège: Mardaga.

Engeström, Y. (1987). Learning by expanding: An activity-theoretical approach to developmental research. Helsinki: Orienta-Konsultit.

Falzon, P. (1994). Les activités méta-fonctionnelles et leur assistance. Le Travail Humain, 57(1), 1-23

Genard, J.-L. (2011). Investiguer le pluralisme de l'agir, SociologieS [En ligne], Grands résumés, L'Action au pluriel. Sociologie des régimes d'engagement, mis en ligne le 06 juillet 2011, consulté le 01 novembre 2012. URL : http://sociologies.revues.org/3574

Giddens, A. (1984). La Constitution de la société, (1984), traduit par M. Audet. Paris: PUF, 2004.

Greimas, A. J. (1966). Sémantique structurale. Paris: Larousse.

Gros, A. (2011). Les formes de l'enquête historique : John Dewey et Max Weber, L'Atelier du Centre de recherches historiques [En ligne], 07 I 2011, mis en ligne le 10 mars 2011, consulté le 01 novembre 2012. URL : http://acrh.revues.org/3668 ; DOI : 10.4000/acrh.3668

Hatchuel, A. (1996). Coopération et conception collective - Variété et crises des rapports de prescription. In de Terssac, G., \& E. Friedberg E. (Eds.), Coopération et conception (pp. 101-121). Toulouse: Octarès Édition.

Hatchuel, A., \& Weil ,B. (2002), La théorie C-K : fondements et usages d'une théorie unifiée de la conception. Colloque sciences de la conception, Lyon, 15-16 mars 2002.

http://www.spatial-computing.org/ michel/lib/exe/fetch.php?media=documents-ro:hatchuelweil2002latheorieck.pdf

Hébert, L. (2006). Le modèle actantiel. In L. Hébert (Ed..), Signo [en ligne], Rimouski (Québec), http://www.signosemio.com (consulté le 11/09/2011).

Hoc, J.-M. (2006). Psychologie cognitive de la planification. Grenoble: Presses Universitaires de Grenoble.

Hutchins, E. (1995), Cognition in the Wild, Cambridge. MA: MIT Press.

Meyerson, (1948). Les fonctions psychologiques et les oeuvres, nouvelle édition (1995). Paris: Albin Michel.

Latour, B. (1996). Petites leçons de sociologie des sciences. Paris: La Découverte

Mead, G. H. (2006). L'esprit, le soi la société (préface et traduction par D. Cefaï \& L. Quéré). Paris: PUF (1934).

Meyerson, I., (1948), Les fonctions psychologiques et les auvres, nouvelle édition (1995). Paris: Albin Michel.

Muniesa, F., \& Callon, M. (2009). La performativité des sciences économiques. In Ph. Steiner \& F. Vatin (Eds.), Traité de sociologie économique (pp. 289-324). Paris: PUF.

Naves, P. ,\& Defalvard, H. (2006). Économie politique de l'action sociale. Paris: Dunod,

Oström, E. (1990), Governing the commons, the evolution of institutions for collective action, Cambridge: Cambridge University Press.

Parsons, T., Bales, R., \& Shils, E. (1953). Working Papers in the Theory of Action. New York: The Free Press.

Peirce, C.S. (2002). Pragmatisme et pragmaticisme, Euvres philosophiques, Volume I, édition établie par et traduction de C. Tiercelin et P. Thibaud, Paris: Éditions du Cerf.

Polanyi, K. (1983). La grande transformation. Paris: Gallimard.

Propp, V. (1970/1928). Morphologie du conte. Paris: Seuil, coll. « Points / Essais », 1970.

Rabardel, P. (1995). Les hommes et les technologies : approche cognitive des instruments contemporains. Paris: Armand Colin.

Rasmussen, J. (1983). Skills, rules, knowledge; signals, signs, and symbols, and other distinctions in human performance models. IEEE Transactions on Systems, Man and Cybernetics, 13, 257-266.

Reynaud J.-D. (1989). Les Règles du jeu : L'action collective et la régulation sociale, Paris : Armand Colin.

Renault, M. (2004). La nécessité d'une approche cognitive de la firme pour une économie fondée sur la connaissance. In M.Baslé \& M.Renault (Eds.), L'économie fondée sur la connaissance-Questions autour du projet européen (pp. 143-182). Paris: Economica. 
Renault, M. (2013). Perspectives, transactions et espaces : une approche transactionnelle appliquée de l'action. Document de travail de l'Université de Rennes 1.

Robichaud, D. (2006). Steps Toward a Relational View of Agency. In F. Cooren, J. R. Taylor, \& E. J. Van Every (Eds.) (2006). Communication as organizing: Empirical and Theoretical Explorations In the dynamic of text and conversation (pp.). Mahwah, NJ: Lawrence Erlbaum Associates.

Schmidt, K., \& Simone, C. (1996). Coordination mechanisms: Towards a conceptual foundation of CSCW systems design. CSCW Journal, 5(2-3), 155-200.

Simpson, B. (2013). Le pragmatisme, Mead et le tournant pratique. Activité, 10(1), dans ce numéro. Taylor, J. R., \& E. J. Van Every (2000). The Emergent Organization: Communication as its Site and Surface. Mahwah, NJ: Lawrence Erlbaum Associates.

Simon, H.A. (1973). De la rationalité substantive à la rationalité procédurale, en ligne, http://www.mcxapc.org/fileadmin/docs/lesintrouvables/simon5.pdf (consulté le 31/10/2012)

Steiner, P. (2008). Sciences cognitives, tournant pragmatique et horizons pragmatistes. Tracés, 15, 85105.

Steiner, P. (2013). C.S. Peirce and Artificial Intelligence: Historical Heritage and (New) Theoretical Stakes. In V.C. Müller (Ed). Philosophy and Theory of Artificial Intelligence (pp. 265-276). SAPERE 5 .

Strauss, A. (1993). Continual Permutations of Action. New York: Aldine de Gruyter.

Suchman, L. (1987). Plans and Situated Actions: The Problem of Human-Machine Communication. New York: Cambridge University Press.

Taylor, J.R., \& Van Every, E.J. (2000). The emergent organization: Communication as its site and surface. Mahwah, NJ: Lawrence Erlbaum Associates.

Theureau, J. (2004). Le cours d'action. Méthode élémentaire. Toulouse: Octarès Édition.

Thévenot, L. (2006). L'action au pluriel : sociologie des régimes d'engagement. Paris: La Découverte.

Terssac, G. de (2002). Le travail : une aventure collective. Toulouse: Octarès éditions.

Terssac, G. de, \& Maggi, B. (1996). Autonomie et conception. In G. de Terssac, \& E. Friedberg E. (Eds.). Coopération et conception (pp.). Toulouse: Octarès Édition.

Tertre du, C. (2005). Services immatériels et relationnels : intensité du travail et santé. @ ctivités, 2(1), 37-49, http://www .activites.org/v2n1/dutertre.pdf

Weber, M. (1978). Economy and Society: An outline of interpretive sociology. University of California Press: New Ed.

Zacklad, M. (1993). Principes de modélisation qualitative pour l'aide à la décision dans les organisations. Thèse de doctorat, Spécialité Contrôle des Systèmes, Compiègne, Université de Technologie de Compiègne.

Zacklad, M. (2000). La théorie des Transactions Intellectuelles : une approche gestionnaire et cognitive pour le traitement du COS, Intellectica 2000/1, 30, 195-222.

Zacklad, M. (2005). Innovation et création de valeur dans les communautés d'action : les transactions communicationnelles symboliques. In R. Teulier \& P. Lorino (Eds.), Entre connaissance et organisation : l'activité collective (pp.). Paris: La Découverte.

Zacklad, M. (2007a). Une théorisation communicationnelle et documentaire des TIC. In B. Reber, \& C. Brossaud (Eds.), Humanités numériques. Nouvelles technologies cognitives et concepts des sciences sociales (pp. 20-35). Londres-Paris: Hermes Publishing.

Zacklad, M. (2007b). Management of the knowing and the known in transactional theory of action. In R. Day, R., \& C. McInerney (Eds.), From Knowledge Management to Knowledge Processes (pp. 301329). Springer-Verlag.

Zacklad, M. (2010a). Sémiotique de la création de valeur dans l'économie des transactions coopératives. In A. Hatchuel, O, Favereau, O., \& F. Aggeri (Eds), L'activité marchande sans le marché (pp. 265283). Paris: Presses de l'École des Mines.

Zacklad, M. (2010b). Introduction aux Appareils de Capture Sémantique dans les TIC et le SI. In J.M. Noyer J.M., \& B. Juanals (Eds.), Technologies de l'Information et Intelligences collectives (pp. 175199). Paris: Hermes-Lavoisier.

Zacklad, M. (2012a). Communication et travail : sortir de l'ambiguïté. CMN 2012 : Communiquer dans 
un monde de normes, Roubaix, France, 7-9 mars.

Zacklad, M. (2012b). Engagement dans les communautés virtuelles et communication transformative. In Actes du colloque «Organisations, performativité et engagement », 80e congrès international ACFAS, Palais des congrès de Montréal, mai 2012, pp. 174-186.

http://www.crpcm.uqam.ca/pages/docs/Actes_colloque_ACFAS_2012.pdf

Zask, J. (2008). Situation ou contexte ? Une lecture de Dewey. Revue internationale de philosophie, $2008 / 3-n^{\circ} 245,313$ à 328.

\section{RESUME}

Dans cet article nous nous utilisons la Sémiotique des Transactions Coopératives pour analyser le travail management que nous définirons comme une activité de cadrage des transactions coopératives qui peut concerner des opérateurs spécialisés, les managers ou les cadres, mais qui plus généralement concerne tous les membres de l'entreprise en tant qu'ils s'impliquent un travail de management, parfois qualifié de travail de régulation (Reynaud, 1989), travail d'organisation (de Terssac, 2002), travail d'articulation (Strauss, 1993) ou d'activité méta-fonctionnelle (Falzon, 1994). Nous commencerons par présenter les principales catégories de la sémiotique des transactions coopérative qui se positionne comme un cadre d'analyse de l'action et de la pratique au niveau «méso», encore en développement, mais comparable à ceux proposés dans d'autres théories en sciences cognitives, en psychologie, en sociologie, en sciences de l'information et de la communication, voire dans certaines approches de l'anthropologie ou de la philosophie des techniques. Pour faire le lien entre l'analyse «micro » des interactions médiatisée et les processus d'organisation et de création de valeur appréhendés à des niveaux plus « macro », nous insisterons plus particulièrement dans cet article sur l'approche pragmatiste des notions de situation et de contexte qui nous permettront de rendre compte de cette dimension essentielle de l'action collective que sont les activités de cadrage. Nous illustrerons ces notions par la présentation d'un cas correspondant à la problématique d'une conduite de changement dans un milieu universitaire visant à introduire un contrôle gestionnaire renforcé inscrit dans la loi sur la réforme des universités françaises. Ce cas sera interprété en termes de cadrage, de conflit de cadrage, de recadrage du contexte transactionnel contribuant à la conduite du changement et à la résistance au changement.

\section{MOTS-CLEFS}

analyse de l'activité, travail de management, transaction, sémiotique, pragmatisme

\section{REFERENCEMENT}

Zacklad, M. (2013). Le travail de management en tant qu'activité de cadrage et de recadrage du contexte des transactions coopératives. Activités, 10(1), 192-220, http://www .activites.org/v10n1/v10n1.pdf

Article soumis le 29 septembre, accepté pour publication le 7 janvier 2013 